\title{
THE MID-INFRARED SPECTRUM OF STAR-FORMING GALAXIES: GLOBAL PROPERTIES OF POLYCYCLIC AROMATIC HYDROCARBON EMISSION
}

\author{
J. D. T. Smith, ${ }^{1}$ B. T. Draine, ${ }^{2}$ D. A. Dale, ${ }^{3}$ J. Moustakas, ${ }^{1}$ R. C. Kennicutt, JR., ${ }^{1,4}$ G. Helou,,${ }^{5}$ L. Armus, ${ }^{6}$ \\ H. Roussel, ${ }^{7}$ K. Sheth, ${ }^{3}$ G. J. Bendo,${ }^{8}$ B. A. Buckalew, ${ }^{6}$ D. Calzetti,,${ }^{9}$ C. W. Engelbracht, ${ }^{1}$ \\ K. D. Gordon, ${ }^{1}$ D. J. Hollenbach, ${ }^{10}$ A. Li, ${ }^{11}$ S. Malhotra, ${ }^{12}$ \\ E. J. Murphy, ${ }^{13}$ And F. Walter ${ }^{7}$ \\ Received 2006 May 5; accepted 2006 October 29
}

\begin{abstract}
We present a sample of low-resolution 5-38 $\mu \mathrm{m}$ Spitzer IRS spectra of the inner few square kiloparsecs of 59 nearby galaxies spanning a large range of star formation properties. A robust method for decomposing mid-infrared galaxy spectra is described and used to explore the behavior of PAH emission and the prevalence of silicate dust extinction. Evidence for silicate extinction is found in $\sim \frac{1}{8}$ of the sample, at strengths that indicate that most normal galaxies undergo $A_{V} \lesssim 3 \mathrm{mag}$ averaged over their centers. The contribution of PAH emission to the total infrared power is found to peak near $10 \%$ and extend up to $\sim 20 \%$ and is suppressed at metallicities $Z \lesssim Z_{\odot} / 4$, as well as in low-luminosity AGN environments. Strong interband PAH feature strength variations ( $2-5$ times) are observed, with the presence of a weak AGN and, to a lesser degree, increasing metallicity shifting power to the longer wavelength bands. A peculiar PAH emission spectrum with markedly diminished 5-8 $\mu \mathrm{m}$ features arises among the sample solely in systems with relatively hard radiation fields harboring low-luminosity AGNs. The AGNs may modify the emitting grain distribution and provide the direct excitation source of the unusual PAH emission, which cautions against using absolute PAH strength to estimate star formation rates in systems harboring active nuclei. Alternatively, the low star formation intensity often associated with weak AGNs may affect the spectrum. The effect of variations in the mid-infrared spectrum on broadband infrared surveys is modeled and points to more than a factor of 2 uncertainty in results that assume a fixed PAH emission spectrum, for redshifts $z=0-2.5$.
\end{abstract}

Subject headings: galaxies: active — galaxies: ISM — infrared: galaxies — techniques: spectroscopic

Online material: machine-readable tables

\section{INTRODUCTION}

The mid-infrared (MIR) spectrum of star-forming galaxies is dominated by strong emission features generally attributed to polycyclic aromatic hydrocarbons (PAHs). PAHs are aromatic molecules ubiquitous in the interstellar medium (ISM) of our own Galaxy and found in most nearby galaxies with ongoing or recent star formation. These large molecules, typically a few $\AA$ in diameter and harboring up to a few hundred carbon atoms, are now commonly believed to be responsible for the prominent suite of broad emission bands in the MIR between 3 and $19 \mu \mathrm{m}$ (Leger \& Puget 1984; Allamandola et al. 1985). PAH molecules efficiently absorb ultraviolet and optical photons and in many environments supply the bulk of the photoelectrons responsible for heating interstellar gas (e.g., Weingartner \& Draine 2001).

The MIR PAH bands can be very strong, compared to both their underlying dust grain continuum and even the reprocessed

\footnotetext{
1 Steward Observatory, University of Arizona, Tucson, AZ; jdsmith@as .arizona.edu.

2 Department of Astrophysical Sciences, Princeton University, Princeton, NJ.

3 Department of Physics and Astronomy, University of Wyoming, Laramie, WY.

4 Institute of Astronomy, Cambridge University, Cambridge, UK.

5 California Institute of Technology, Pasadena, CA.

6 Spitzer Science Center, Caltech, Pasadena, CA.

7 Max-Planck-Institut für Astronomie, Heidelberg, Germany.

8 Imperial College, Blackett Laboratory, London, UK.

9 Space Telescope Science Institute, Baltimore, MD.

10 NASA Ames Research Center, Moffett Field, CA.

11 Department of Physics and Astronomy, University of Missouri, Columbia, MO.

12 Department of Physics and Astronomy, Arizona State University, Tempe, AZ.

13 Department of Astronomy, Yale University, New Haven, CT.
}

bolometric emission of larger dust grains. In galaxies with intense star formation, up to $20 \%$ of the total infrared (TIR) luminosity is emitted in the PAH bands alone, and, longward of the Lyman and Balmer breaks, they are among the most prominent spectral features with sufficient contrast for photometric redshift determination. In deep infrared surveys, they can induce peaks in the flux-limited number density of 15-24 $\mu$ m-detected galaxies at redshifts $z \gtrsim 1$, as they move through fixed filter passbands (e.g., Barvainis et al. 1999; Le Floc'h et al. 2005; Caputi et al. 2006). Thus, they are of fundamental importance in modeling deep infrared survey results and especially in constraining the role of dust-obscured star formation at early epochs. Yet the behavior or even presence of PAHs in the distant universe is largely unexplored. Despite tantalizing indications of PAH emission at redshift beyond $z \gtrsim 2$ (Yan et al. 2005; Lutz et al. 2005; Huang et al. 2006), other surveys probing the MIR properties of optically faint distant galaxy samples have uncovered MIR spectra dominated by continuum emission, cut by deep silicate absorption (Houck et al. 2005).

A prevailing difficulty in studying PAH emission at high redshifts is the relative ambiguity of the 7-13 $\mu \mathrm{m}$ spectrum of starforming galaxies, with the broad PAH features at 7.7 and $11.3 \mu \mathrm{m}$ almost perfectly bracketing the $10 \mu \mathrm{m}$ silicate absorption trough. Silicate absorption can be pronounced in some galaxies' spectra, including high-redshift objects dominated by active galactic nucleus (AGN) emission. At low signal, or in broad photometric bands, discriminating between these two physically distinct but observationally similar signatures can be challenging. Interpretation of these spectral signatures, as well as of broadband properties of even fainter and more distant systems, has relied predominantly 
on a small set of local template spectra developed primarily from Infrared Space Observatory (ISO) observations of bright nearby systems.

The Spitzer Infrared Nearby Galaxies Survey (SINGS; Kennicutt et al. 2003) provides an exceptional sample in which to explore the global and spatially resolved behavior of these fundamental emission bands over a much broader range of physical environments than has been possible before. We focus here on the global behavior of the PAH emission spectrum and its underlying continuum. A future paper will focus on the spatially resolved behavior of PAH emission in the centers and extended disks of galaxies in the SINGS sample.

\section{BACKGROUND ON THE EMISSION PROCESSES}

The "unidentified infrared bands" were first discovered in 1973 in the spectra of Galactic planetary nebulae by Gillett et al. (1973). PAHs were later proposed as the origin of these features (Leger \& Puget 1984; Allamandola et al. 1985) and invoked to explain the $12 \mu \mathrm{m}$ infrared excess seen by the Infrared Astronomical Satellite (IRAS) in the diffuse cirrus of the Milky Way (Puget et al. 1985). Although the carriers of the MIR emission bands are now commonly attributed to the broad class of PAH molecules, other interpretations remain consistent with at least some aspects of the bands' strength, shape, and location (see Li \& Draine 2001 and references therein). Although the only certainty is that they arise primarily in material with $s p^{2}$ hybridized carbon-carbon bonds by infrared fluorescence, we adopt the convention of referring to them as PAH emission bands; it should be kept in mind that the emitters could encompass a larger group of individual species. In this same spirit, we refer to PAHs interchangeably as both molecules and small grains.

The ISO Key Project on Nearby Galaxies (Helou et al. 2000) explored the $2-11 \mu \mathrm{m}$ spectrum of a sample of normal galaxies. ISO was limited to relatively high surface brightness sources, whereas SINGS extends to much lower luminosity and surface brightness and is exploring a much broader range of local interstellar environment. A surprising result of the Key Project was the relative uniformity of the PAH emission spectrum over a range of different galaxy environments. Lu et al. (2003) found band-to-band variations of at most $15 \%$ out to $11 \mu \mathrm{m}$. A simple physical argument that can be invoked to explain this apparent uniformity relates to the underlying emission mechanism of small grains. Since PAH grains are transiently excited by single photons to high temperatures ( higher than their equilibrium temperatures; see Leger \& Puget 1984; Draine \& Anderson 1985; Allamandola et al. 1985; Draine \& Li 2001), the emission spectrum produced by a single grain population is relatively insensitive to the strength and hardness of the exciting radiation field (Leger \& Puget 1984), and the resulting PAH spectrum might therefore be expected to be quite uniform. However, a variety of mechanisms are known to affect to various degrees the strength of the individual PAH bands, including ionization state, distribution of grain sizes, dehydrogenation, and multiphoton heating in intense radiation fields (for a review see Li 2004). The possible destruction mechanisms of PAH grains are varied as well, including photo-thermo (Léger et al. 1989; Guhathakurta \& Draine 1989) and Coulomb dissociation, sputtering, and dissociation in shocks.

Evidence in our own Galaxy (e.g., Joblin et al. 1996; Verstraete et al. 1996; Hony et al. 2001; Peeters et al. 2004; Bregman \& Temi $2005)$ and in resolved regions in nearby galaxies like the LMC and SMC (Contursi et al. 2000; Vermeij et al. 2002) indicates that the PAH emission spectrum is variable on small scales and can depend sensitively on the local gas density and details of the in- cident radiation field. Variations can occur in the relative strengths, widths, and wavelength centers of individual emission bands (e.g., Peeters et al. 2002).

It is not yet known whether the primary processes regulating PAH emission are chemical, mechanical, or radiative, or whether they are expressed through inhibited or enhanced grain formation rates, selective destruction in differing environments, or varying efficiencies of photoexcitation. Given the predicted and observed diversity in the form of the PAH emission spectrum within individual objects in the Galaxy, the question that remains and is explored here is whether the natural variations found on small scales vanish when averaged over real distributions of emitting environments in the disks and nuclei of galaxies.

\section{OBSERVATIONS AND REDUCTION}

SINGS includes uniform 5-38 $\mu \mathrm{m}$ spectral mapping with the Infrared Spectrograph (IRS; Houck et al. 2004). Full observational details regarding the SINGS spectral mapping program can be found in Kennicutt et al. (2003). Briefly, a low-resolution $(R \sim 50-100), 5-15 \mu \mathrm{m}$ spectral map of approximate size $55^{\prime \prime} \times$ $34^{\prime \prime}$ is centered on the nucleus of each target. Larger, 15-38 $\mu \mathrm{m}$ radial strip maps extending to roughly half the optical radius and covering $50^{\prime \prime} \times 3.5^{\prime}-15.4^{\prime}$ are also obtained. The total effective integration time per pixel was uniform, at $28 \mathrm{~s}$ in the short-wavelength module and 2 minutes in the long-wavelength module. The IRS spectra were supplemented for all sample targets with scan map images at 24, 70, and $160 \mu \mathrm{m}$ obtained with the MIPS instrument (Rieke et al. 2004).

\subsection{Sample}

A subset of 59 galaxies from the SINGS sample was selected, and their relevant parameters are given in Table 1. The subsample spans a range in luminosity and infrared/optical ratio similar to the complete sample and includes galaxies from all represented morphological types. The only requirements for inclusion in the subsample were full 5-38 $\mu \mathrm{m}$ spectral coverage and sufficient signal-to-noise ratio $(\mathrm{S} / \mathrm{N})$ to put useful upper limits on PAH emission (the continuum must be detectable). Among the galaxies not included were an individual source with anomalous silicate extinction (Roussel et al. 2006), a source with strong silicate emission, and several undetected low-luminosity dwarf galaxies.

\subsection{MIPS Data Reduction and Matched Fluxes}

All MIPS images were reduced as described in Dale et al. (2005), and global MIPS fluxes were taken from that work, modified slightly $(<10 \%)$ to account for the most recent MIPS calibration factors. Obtaining total infrared luminosities $\left(L_{\mathrm{TIR}}\right)$ matched to the apertures listed in Table 1, which are in most cases smaller than the $38^{\prime \prime}$ diffraction beam full width of the MIPS $160 \mu \mathrm{m}$ instrument, required a method to overcome the inherent resolution limits of the two long-wavelength channels. We adopted a consistent approach to estimate the 70 and $160 \mu \mathrm{m}$ surface brightness. Representative $24 \mu \mathrm{m}: 70 \mu \mathrm{m}$ : $160 \mu \mathrm{m}$ band ratios were obtained in $25^{\prime \prime}$ radius regions centered on the spectral extraction apertures, from 24 and $70 \mu \mathrm{m}$ images convolved to the MIPS $160 \mu \mathrm{m}$ resolution, and from $160 \mu \mathrm{m}$ images at native resolution. These central color ratios were assumed to hold in the smaller spectral apertures and were combined with matched $24 \mu \mathrm{m}$ photometry (which does not suffer resolution effects) to predict absolute 70 and $160 \mu \mathrm{m}$ surface brightness. From these, the matched total infrared intensity, $I_{\mathrm{TIR}}$, was calculated according to the prescription of Dale \& Helou (2002). Except where otherwise noted, all references to the total infrared luminosity are matched in this way to the spectral apertures. 
TABLE 1

Sample Parameters

\begin{tabular}{|c|c|c|c|c|c|c|c|c|c|c|}
\hline \multirow[b]{2}{*}{$\begin{array}{c}\text { GALAXY } \\
\text { (1) }\end{array}$} & \multirow[b]{2}{*}{$\begin{array}{c}\text { Nuclear TyPe } \\
\text { (2) }\end{array}$} & \multirow[b]{2}{*}{$\begin{array}{c}\text { Distance } \\
(\mathrm{Mpc}) \\
(3)\end{array}$} & \multicolumn{5}{|c|}{ EXTRACTION APERTURE } & \multirow[b]{2}{*}{$\begin{array}{c}L_{\mathrm{TIR}} \\
\left(10^{8} L_{\odot}\right) \\
(9)\end{array}$} & \multirow[b]{2}{*}{$\begin{array}{l}f_{\text {TIR }} \\
(10)\end{array}$} & \multirow[b]{2}{*}{$\begin{array}{c}L_{\mathrm{TIR}} / \nu L_{\nu}(B) \\
\quad(11)\end{array}$} \\
\hline & & & $\begin{array}{l}\text { R.A. } \\
\text { (4) }\end{array}$ & $\begin{array}{c}\text { Decl. } \\
(5)\end{array}$ & $\begin{array}{c}\text { Size } \\
(\operatorname{arcsec}) \\
(6)\end{array}$ & $\begin{array}{c}A \\
\left(\mathrm{kpc}^{2}\right) \\
(7)\end{array}$ & $\begin{array}{c}\text { P.A. } \\
\text { (deg) } \\
(8)\end{array}$ & & & \\
\hline Ho II... & $\mathrm{H}_{\mathrm{II}}$ & 3.4 & 081912.86 & +704309.6 & $14.8 \times 9.3$ & 0.04 & -155.6 & 0.1 & 0.06 & 1.94 \\
\hline 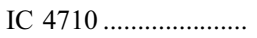 & $\mathrm{H}$ II & 9.0 & 182839.55 & -665820.8 & $11.1 \times 9.2$ & 0.19 & 176.9 & 0.3 & 0.06 & 3.22 \\
\hline Mrk $33 \ldots \ldots \ldots \ldots \ldots \ldots$ & $\mathrm{H}_{\text {II }}$ & 22.9 & 103231.82 & +542402.5 & $38.8 \times 31.4$ & 15.03 & 41.9 & 51.8 & 0.78 & 1.88 \\
\hline 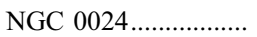 & $\mathrm{H}_{\text {II }}$ & 7.3 & 000956.37 & -245751.2 & $35.1 \times 29.6$ & 1.32 & -39.5 & 0.9 & 0.17 & 0.76 \\
\hline 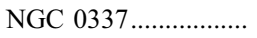 & $\mathrm{H}_{\text {II }}$ & 22.4 & 005950.20 & -073445.8 & $42.6 \times 25.9$ & 12.99 & 149.0 & 60.4 & 0.37 & 3.40 \\
\hline NGC $0584 \ldots \ldots$ & LINER & 20.0 & 013120.90 & -065205.1 & $18.5 \times 18.5$ & 3.20 & -32.5 & 0.7 & 0.12 & 0.03 \\
\hline NGC $0628 \ldots . .$. & $\mathrm{H}$ II & 7.3 & 013641.60 & +154700.0 & $53.6 \times 29.6$ & 1.99 & 151.8 & 2.7 & 0.03 & 0.90 \\
\hline 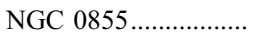 & $\mathrm{H}$ II & 9.6 & 021403.70 & +275238.4 & $18.5 \times 24.1$ & 0.95 & 156.1 & 2.1 & 0.55 & $\ldots$ \\
\hline 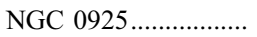 & $\mathrm{H}$ II & 9.1 & 022717.25 & +33 3441.6 & $27.8 \times 29.6$ & 1.61 & 156.2 & 2.3 & 0.05 & 1.17 \\
\hline NGC 1097................ & LINER & 17.1 & 024618.86 & -301627.2 & $37.0 \times 31.4$ & 7.99 & 146.7 & 249.3 & 0.38 & 6.19 \\
\hline 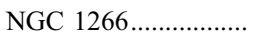 & LINER & 30.0 & 031600.71 & -022536.9 & $22.2 \times 20.4$ & 9.58 & 157.7 & 141.9 & 0.54 & 16.24 \\
\hline 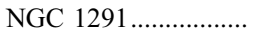 & LINER & 10.8 & 031718.59 & -410628.0 & $38.8 \times 27.7$ & 2.97 & -78.5 & 4.2 & 0.11 & 0.17 \\
\hline 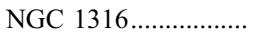 & LINER & 24.3 & 032241.68 & -371229.4 & $22.2 \times 25.9$ & 7.99 & 149.0 & 27.7 & 0.28 & 0.27 \\
\hline 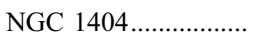 & LINER & 18.5 & 033851.95 & -353539.1 & $25.9 \times 22.2$ & 4.60 & -79.7 & 0.6 & 0.18 & 0.02 \\
\hline 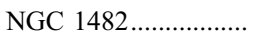 & H II & 23.2 & 035438.88 & -203007.1 & $27.7 \times 29.6$ & 10.40 & -171.4 & 299.2 & 0.68 & 38.04 \\
\hline 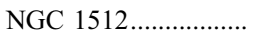 & LINER & 11.8 & 040354.17 & -432054.4 & $22.2 \times 25.9$ & 1.89 & 141.9 & 8.7 & 0.21 & 1.62 \\
\hline 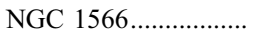 & Seyfert & 20.3 & 042000.33 & -545616.6 & $25.9 \times 24.1$ & 6.06 & 129.0 & 47.2 & 0.08 & 2.06 \\
\hline 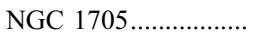 & $\mathrm{H}$ II & 5.1 & 045414.50 & -532136.4 & $14.8 \times 14.8$ & 0.13 & 120.9 & 0.1 & 0.19 & 0.41 \\
\hline 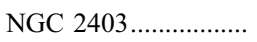 & $\mathrm{H}_{\mathrm{II}}$ & 3.2 & 073649.95 & +653603.5 & $51.8 \times 33.3$ & 0.42 & 30.4 & 0.8 & 0.02 & 0.62 \\
\hline 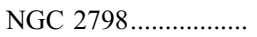 & $\mathrm{H}$ II & 26.2 & 091722.80 & +415959.4 & $25.9 \times 22.2$ & 9.26 & 23.2 & 246.4 & 0.66 & 6.69 \\
\hline 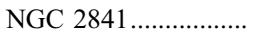 & Seyfert & 14.1 & 092202.50 & +505834.1 & $22.2 \times 22.2$ & 2.30 & 38.6 & 4.0 & 0.03 & 0.11 \\
\hline 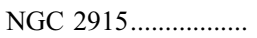 & H II & 3.8 & 092610.03 & -763732.2 & $18.5 \times 18.5$ & 0.11 & 8.4 & 0.1 & 0.33 & $\ldots$ \\
\hline 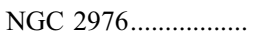 & $\mathrm{H}$ II & 3.6 & 094715.22 & +675500.3 & $38.8 \times 27.8$ & 0.32 & 32.5 & 0.7 & 0.08 & 0.93 \\
\hline 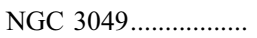 & $\mathrm{H}_{\text {II }}$ & 23.9 & 095449.59 & +09 1618.1 & $37.0 \times 20.4$ & 10.11 & 15.1 & 32.7 & 0.61 & 2.18 \\
\hline 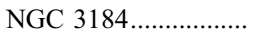 & $\mathrm{H}_{\mathrm{II}}$ & 11.1 & 101816.90 & +412524.7 & $14.8 \times 13.0$ & 0.56 & 33.9 & 2.8 & 0.03 & 1.28 \\
\hline 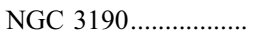 & LINER & 20.9 & 101805.63 & +214954.2 & $27.7 \times 31.5$ & 8.96 & 18.5 & 30.9 & 0.37 & 0.58 \\
\hline 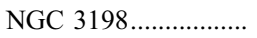 & LINER & 13.7 & 101954.84 & +453258.7 & $22.2 \times 22.2$ & 2.17 & 36.0 & 15.6 & 0.17 & 5.46 \\
\hline 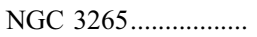 & $\mathrm{H}$ II & 23.2 & 103106.80 & +284745.6 & $27.8 \times 22.2$ & 7.76 & 22.1 & 25.2 & 0.71 & 1.81 \\
\hline 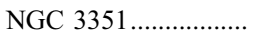 & $\mathrm{H}$ II & 9.3 & 104357.72 & +114213.5 & $40.7 \times 29.6$ & 2.46 & -162.3 & 28.5 & 0.36 & 3.20 \\
\hline 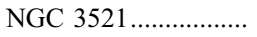 & LINER & 10.1 & 110548.58 & -000207.3 & $53.7 \times 31.4$ & 4.01 & -162.1 & 28.3 & 0.10 & 1.21 \\
\hline 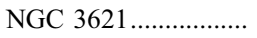 & LINER & 6.6 & 111816.51 & -324849.3 & $53.6 \times 31.4$ & 1.75 & -145.9 & 6.1 & 0.07 & 1.71 \\
\hline 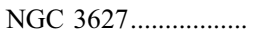 & Seyfert & 9.4 & 112015.04 & +125929.0 & $44.4 \times 31.5$ & 2.89 & 22.8 & 23.5 & 0.09 & 0.82 \\
\hline 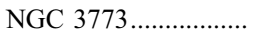 & $\mathrm{H}$ II & 11.9 & 113812.98 & +120645.8 & $24.1 \times 24.0$ & 1.92 & -159.6 & 4.1 & 0.53 & 0.82 \\
\hline 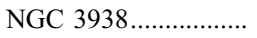 & LINER & 13.3 & 115249.32 & +440713.6 & $49.9 \times 29.6$ & 6.19 & 36.6 & 11.2 & 0.10 & 0.75 \\
\hline 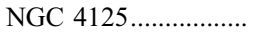 & LINER & 22.9 & 120805.84 & +65 1029.5 & $24.0 \times 24.1$ & 7.13 & 173.4 & 5.1 & 0.32 & 0.06 \\
\hline 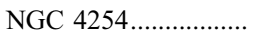 & $\mathrm{H}$ II & 16.6 & 121849.57 & +142457.5 & $40.7 \times 24.1$ & 6.33 & 18.9 & 56.7 & 0.11 & 1.59 \\
\hline 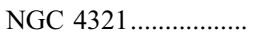 & LINER & 14.3 & 122254.87 & +154919.2 & $31.4 \times 25.9$ & 3.92 & 24.6 & 54.6 & 0.16 & 3.39 \\
\hline 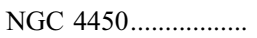 & LINER & 16.6 & 122829.71 & +170508.7 & $48.1 \times 29.6$ & 9.20 & 31.4 & 9.8 & 0.20 & 0.23 \\
\hline NGC $4536 \ldots \ldots \ldots \ldots \ldots . . . . . . .$. & $\mathrm{H}$ II & 14.4 & 123427.03 & +021116.5 & $25.9 \times 24.0$ & 3.06 & 21.4 & 96.3 & 0.73 & 9.12 \\
\hline 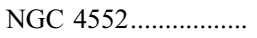 & Seyfert & 15.9 & 123539.88 & +123323.3 & $25.9 \times 24.0$ & 3.71 & -160.9 & 0.7 & 0.25 & 0.01 \\
\hline 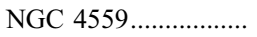 & $\mathrm{H}$ II & 10.3 & 123557.58 & +275734.2 & $51.8 \times 33.3$ & 4.29 & 40.9 & 8.8 & 0.13 & 0.79 \\
\hline 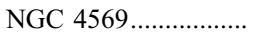 & Seyfert & 16.6 & 123649.76 & +130945.5 & $25.9 \times 22.2$ & 3.72 & 25.4 & 45.3 & 0.31 & $\ldots$ \\
\hline 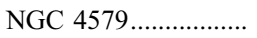 & Seyfert & 16.6 & 123743.53 & +114903.8 & $22.2 \times 18.5$ & 2.65 & 19.6 & 17.1 & 0.12 & 0.45 \\
\hline 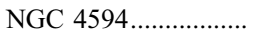 & Seyfert & 9.3 & 123959.56 & -113723.2 & $46.3 \times 31.4$ & 2.98 & 17.4 & 4.3 & 0.10 & 0.08 \\
\hline 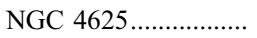 & $\mathrm{H}$ II & 9.2 & 124152.68 & +411626.9 & $37.0 \times 29.6$ & 2.16 & 46.3 & 2.4 & 0.39 & 0.68 \\
\hline 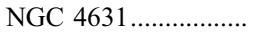 & $\mathrm{H}$ II & 8.1 & 124207.80 & +323234.6 & $50.0 \times 33.3$ & 2.54 & -175.9 & 32.6 & 0.12 & 5.21 \\
\hline 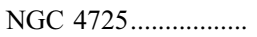 & Seyfert & 11.9 & 125026.59 & +253001.2 & $31.4 \times 20.4$ & 2.13 & 31.2 & 3.6 & 0.04 & 0.28 \\
\hline 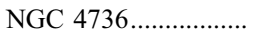 & LINER & 5.0 & 125053.15 & +410714.4 & $53.7 \times 33.3$ & 1.05 & -161.9 & 12.2 & 0.18 & 0.35 \\
\hline NGC 4826................. & Seyfert & 5.0 & 125643.59 & +214058.0 & $29.6 \times 31.5$ & 0.54 & 30.2 & 12.3 & 0.34 & 2.38 \\
\hline 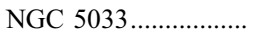 & Seyfert & 14.8 & 131327.32 & +363535.2 & $49.9 \times 31.5$ & 8.11 & 53.1 & 61.4 & 0.24 & 1.95 \\
\hline 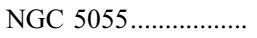 & LINER & 7.8 & 131549.35 & +420145.7 & $38.8 \times 29.6$ & 1.65 & 22.9 & 16.9 & 0.08 & $\ldots$ \\
\hline NGC 5194.................. & Seyfert & 7.8 & 132952.80 & +471143.5 & $55.5 \times 31.5$ & 2.48 & 61.9 & 32.8 & 0.08 & 2.36 \\
\hline NGC $5195 \ldots \ldots \ldots \ldots . . . . . . .$. & Seyfert & 8.0 & 132959.50 & +471556.7 & $33.3 \times 25.9$ & 1.30 & 24.3 & 16.4 & 0.75 & 2.66 \\
\hline 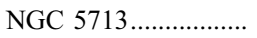 & $\mathrm{H}$ II & 29.4 & 144011.38 & -001724.2 & $44.4 \times 29.6$ & 26.72 & 18.7 & 318.9 & 0.47 & 5.99 \\
\hline NGC $5866 \ldots \ldots \ldots \ldots . . . . . . . .$. & LINER & 15.1 & 150629.48 & +554545.0 & $22.2 \times 29.6$ & 3.54 & 29.4 & 22.9 & 0.41 & 0.59 \\
\hline 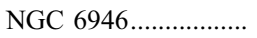 & $\mathrm{H}$ II & 6.8 & 203452.23 & +600914.4 & $44.4 \times 25.9$ & 1.25 & 120.7 & 41.2 & 0.11 & 39.07 \\
\hline 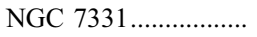 & LINER & 14.5 & 223704.15 & +342455.3 & $55.5 \times 12.9$ & 3.56 & -31.1 & 26.5 & 0.05 & 1.22 \\
\hline 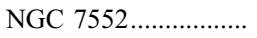 & LINER & 21.0 & 231610.83 & -423505.5 & $37.0 \times 29.6$ & 11.36 & -30.1 & 537.4 & 0.58 & 12.97 \\
\hline 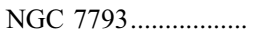 & $\mathrm{H}$ II & 3.8 & 235749.84 & -323527.1 & $53.6 \times 31.4$ & 0.58 & 165.5 & 1.0 & 0.04 & 1.08 \\
\hline
\end{tabular}

Notes.- Units of right ascension are hours, minutes, and seconds, and units of declination are degrees, arcminutes, and arcseconds. Col. (2): Based on the optical

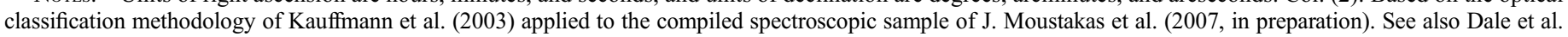

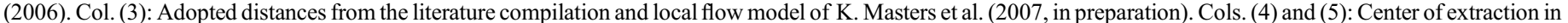

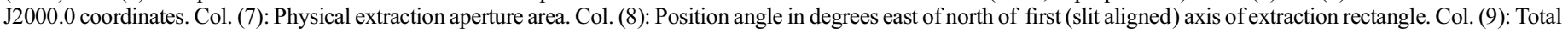

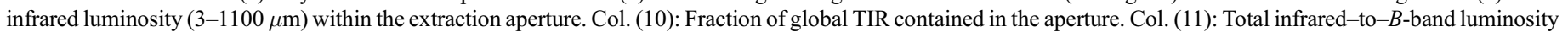
ratio in aperture. 


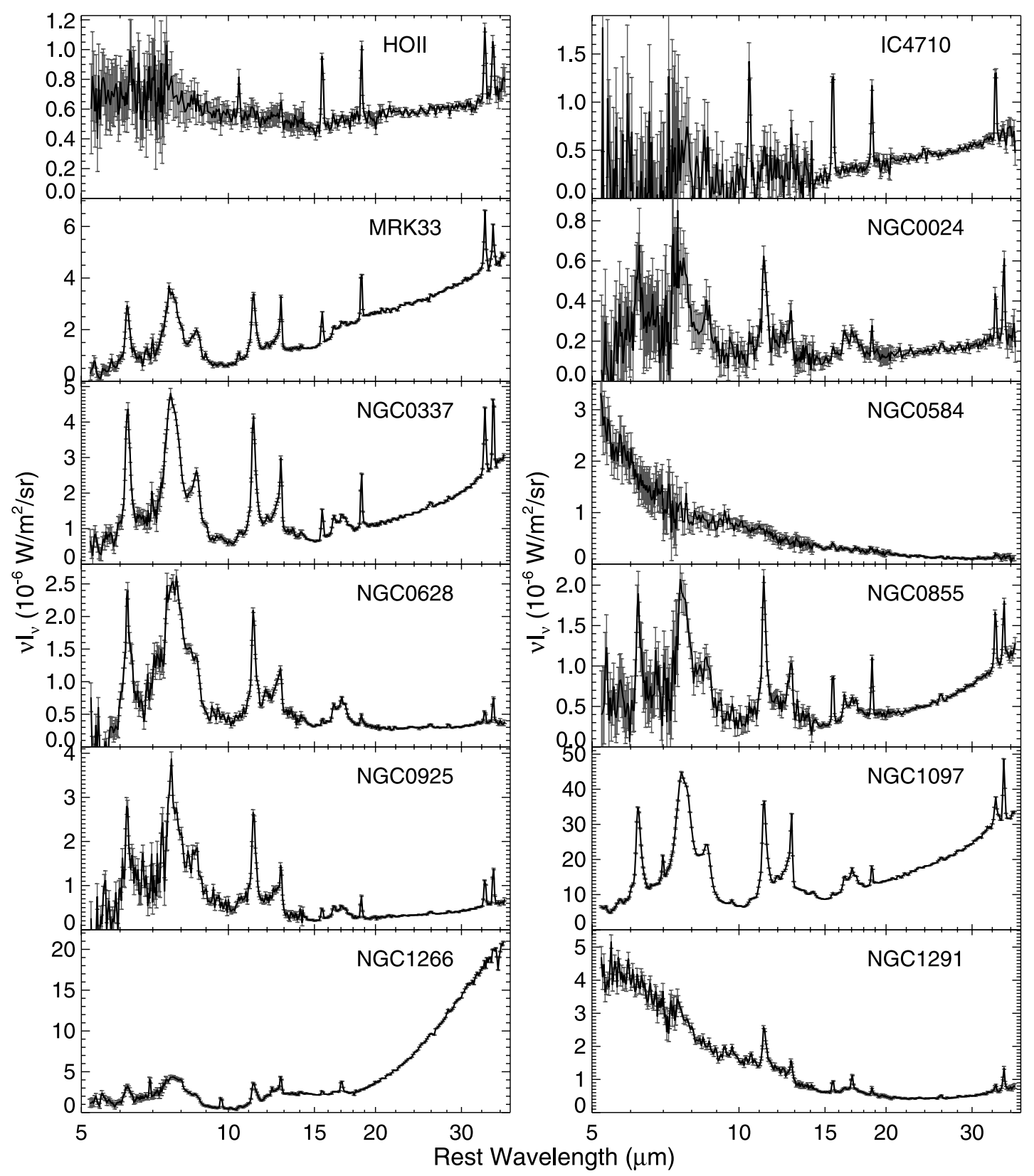

FIG. 1.-Full low-resolution IRS central spectra with logarithmic wavelength axis. Data points are connected.

Since the $70 \mu \mathrm{m} / 24 \mu \mathrm{m}$ and $160 \mu \mathrm{m} / 24 \mu \mathrm{m}$ ratios in galaxies tend to increase as the strength of the radiation field diminishes (e.g., Li \& Draine 2001), this method can overestimate $I_{\text {TIR }}$ if the innermost regions have warmer FIR colors than the surrounding material. To estimate the magnitude of this effect, we considered the nuclear and inner disk MIPS photometry of Dale et al. (2005) from nearby SINGS galaxies M81 and M51. If the luminosityweighted ratio of nuclear to off-nuclear emission in the $25^{\prime \prime}$ beam is $3: 1$, the maximum amount by which $I_{\mathrm{TIR}}$ is overestimated using this technique is $25 \%$; most galaxies should show no bias in this estimate.

\subsection{IRS Data Reduction and Spectra}

All IRS data were reduced with CUBISM (J. D. Smith et al. 2007, in preparation), a custom tool created for the assembly and analysis of spatially resolved spectral cubes from IRS spectral maps. The flux calibration was achieved using appropriate ex- tended source corrections (see J. D. Smith et al. 2007, in preparation). All spectral data were processed with IRS pipeline version S14. Spectra were extracted from IRS modules longlow (LL; 15-37 $\mu \mathrm{m})$ and short-low (SL; 5-15 $\mu \mathrm{m})$ in matched extraction apertures of varying size, chosen to encompass the largest useful region of circumnuclear and inner disk emission, including star-forming rings and central star formation knots. Details of the extraction apertures are given in Table 1. The enclosed fraction of the total infrared emission illustrates the range of aperture coverage: from $5 \%-10 \%$ in large nearby galaxies to nearly the entire system for smaller and more distant targets. Each spectrum is composed of data from the four low-resolution orders of IRS: SL2 (5.25-7.6 $\mu \mathrm{m})$, SL1 (7.5-14.5 $\mu \mathrm{m})$, LL2 (14.5-20.75 $\mu \mathrm{m})$, and LL1 $(20.5-38.5 \mu \mathrm{m})$. Slight mismatches between order segments, resulting from small residual photometric and astrometric uncertainties, were addressed by fitting the continuum near the overlap region with low-order polynomials, 


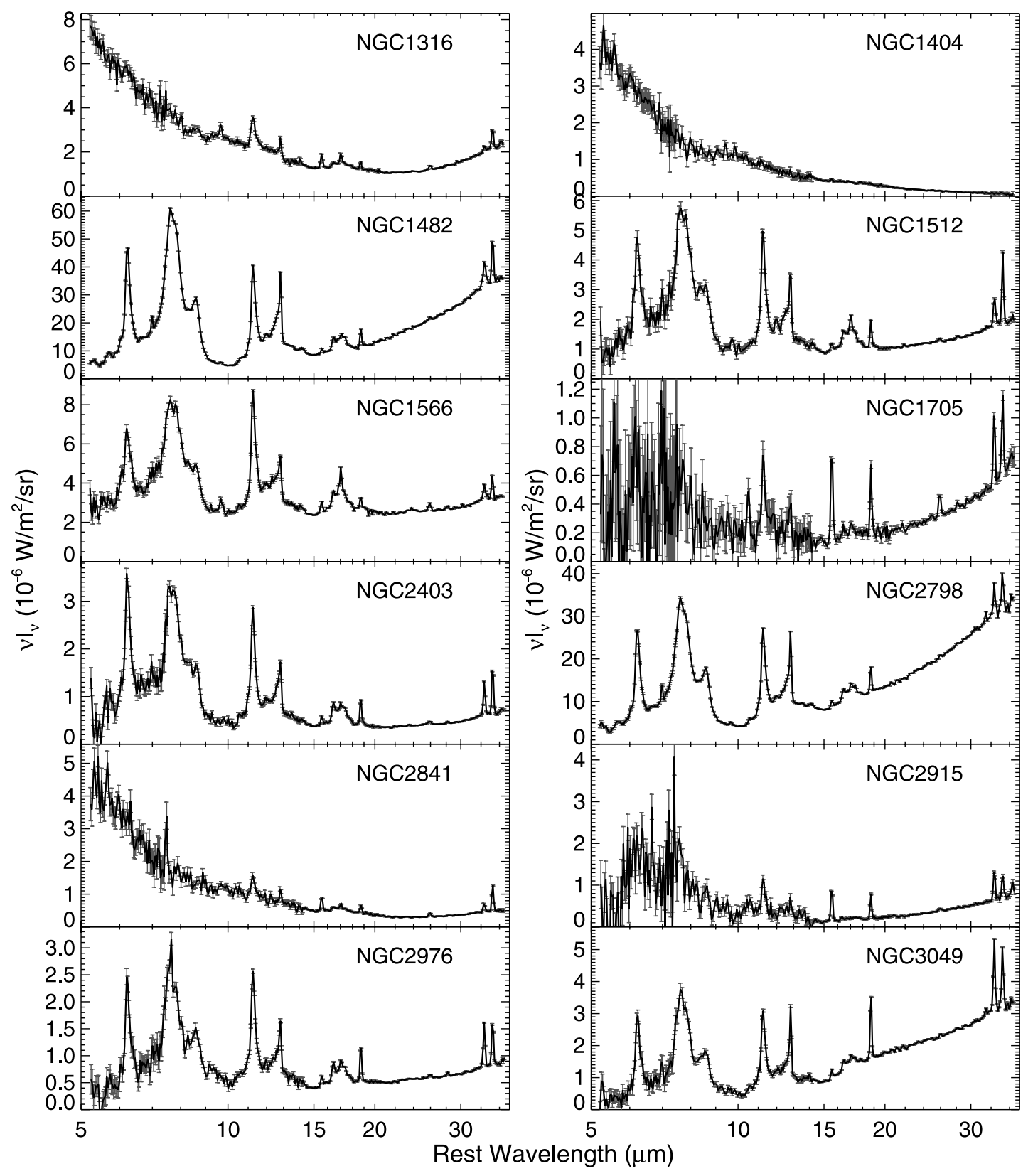

FIG. 1.-Continued

then scaling SL2 to match SL1, LL1 to match LL2, and finally SL to match LL, a method based on cross calibration with IRAC and MIPS. The scaled spectra were then interpolated onto a common wavelength grid and averaged in the overlap region. Typical scaling adjustments were $10 \%$.

Statistical errors are propagated from the ramp-fitting uncertainties produced by the IRS pipeline. Full flux uncertainty cubes are extracted alongside the flux cubes, and the resulting spectral uncertainties are used consistently throughout the analysis. At $\mathrm{S} / \mathrm{N}$ above $\sim 10$, systematic errors exceed the statistical uncertainties in background-subtracted IRS data, particularly in certain wavelength regimes, e.g., 20-22 $\mu \mathrm{m}$, where LL1 residual fringing is strong, and at the ends of the spectral orders, where throughput declines sharply. The magnitude of these systematic errors is typically $15 \%$ or less. All spectral mapping BCD exposures were background subtracted, using background frames taken from dedicated offset observations or from the periphery of the spectral maps themselves. In a small number of cases, when suitable SL background observations were not available, off-source exposures from the archive, observed nearby in time ( $\Delta t<3$ days) and ecliptic latitude $\left(\left|l-l_{0}\right|<10^{\circ}\right)$, were substituted.

All 59 low-resolution IRS spectra are shown in Figure 1. The error bars include only statistical uncertainty, although fixed uncertainties are important at $\mathrm{S} / \mathrm{N} \gtrsim 10$.

\section{SPECTRAL DECOMPOSITION OF THE MIR SPECTRA OF GALAXIES}

In star-forming galaxies, the MIR spectral region hosts among the greatest diversity of emission components of any wavelength regime. The broad, strong PAH features are set against a background continuum transitioning from the rapidly declining direct photospheric emission of old, cool stars at the shorter MIR wavelengths to intermediate wavelengths dominated by hot, stochastically heated small dust grains, settling finally on the 


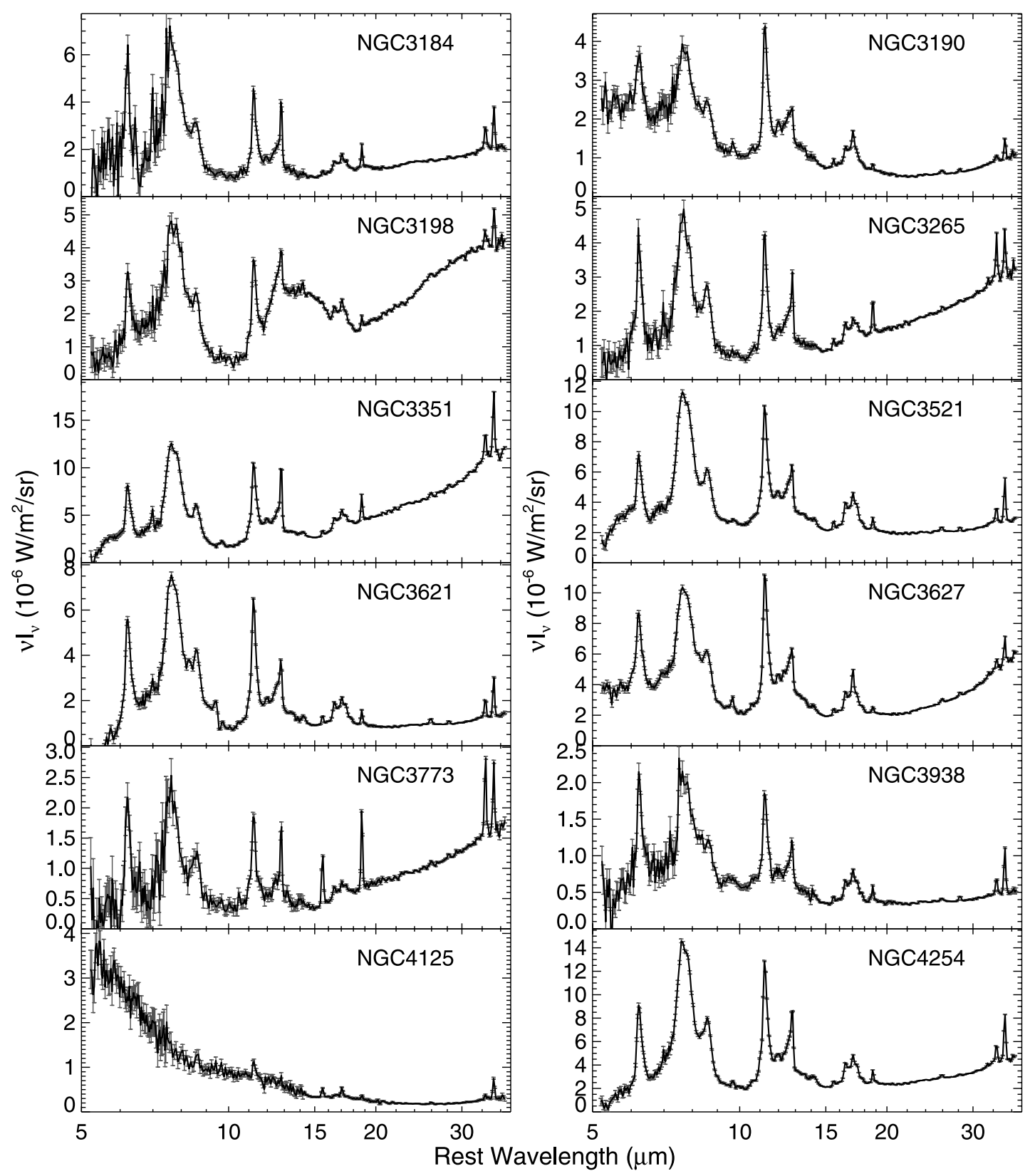

FIG. 1.-Continued

steadily rising emission of the energetically important cool dust component, radiating thermally at longer wavelengths. Set against this diversity of continuum and emission features are broad absorption bands of silicate (at 9.7 and $18 \mu \mathrm{m}$ ) and, in extreme cases, water and other ices (e.g., Spoon et al. 2004). Many of the PAH features are blended with prominent forbidden spectral lines, with [Ne II] $12.8 \mu \mathrm{m}$, blended with a band at $12.7 \mu \mathrm{m}$, being the most severe example. Especially at low spectral resolution, separating the lines, features, multiple continuum components, and absorption features in a consistent way requires a coherent approach.

To decompose low-resolution IRS spectra, we construct a model consisting of a small number of physically motivated components: dust continuum in fixed equilibrium temperature bins, starlight, prominent emission lines, individual and blended $\mathrm{PAH}$ emission bands, and extinction by silicate grains mixed uniformly with all emitting components. The main purpose of this simple yet physically realistic decomposition method is to estimate robustly the strengths of weak features, features blended with line emission, and features affected by moderate silicate absorption, both in absolute terms and relative to the local continuum.

\subsection{The Model}

The surface brightness in the spectral extraction aperture is modeled as a sum of (1) starlight continuum, (2) featureless thermal dust continuum, (3) pure rotational lines of $\mathrm{H}_{2}$, (4) finestructure lines, and (5) dust emission features. The model also allows for dust extinction, characterized by an optical depth $\tau_{\lambda}$ through the emitting region. If the absorbing dust is assumed to be uniformly mixed with the emitting material, the emergent flux intensity is then

$I_{\nu}=\left[\tau_{\star} B_{\nu}\left(T_{\star}\right)+\sum_{m=1}^{M} \tau_{m} \frac{B_{\nu}\left(T_{m}\right)}{\left(\lambda / \lambda_{0}\right)^{2}}+\sum_{r=1}^{R} I_{r}(\nu)\right] \frac{\left(1-e^{-\tau_{\lambda}}\right)}{\tau_{\lambda}}$, 


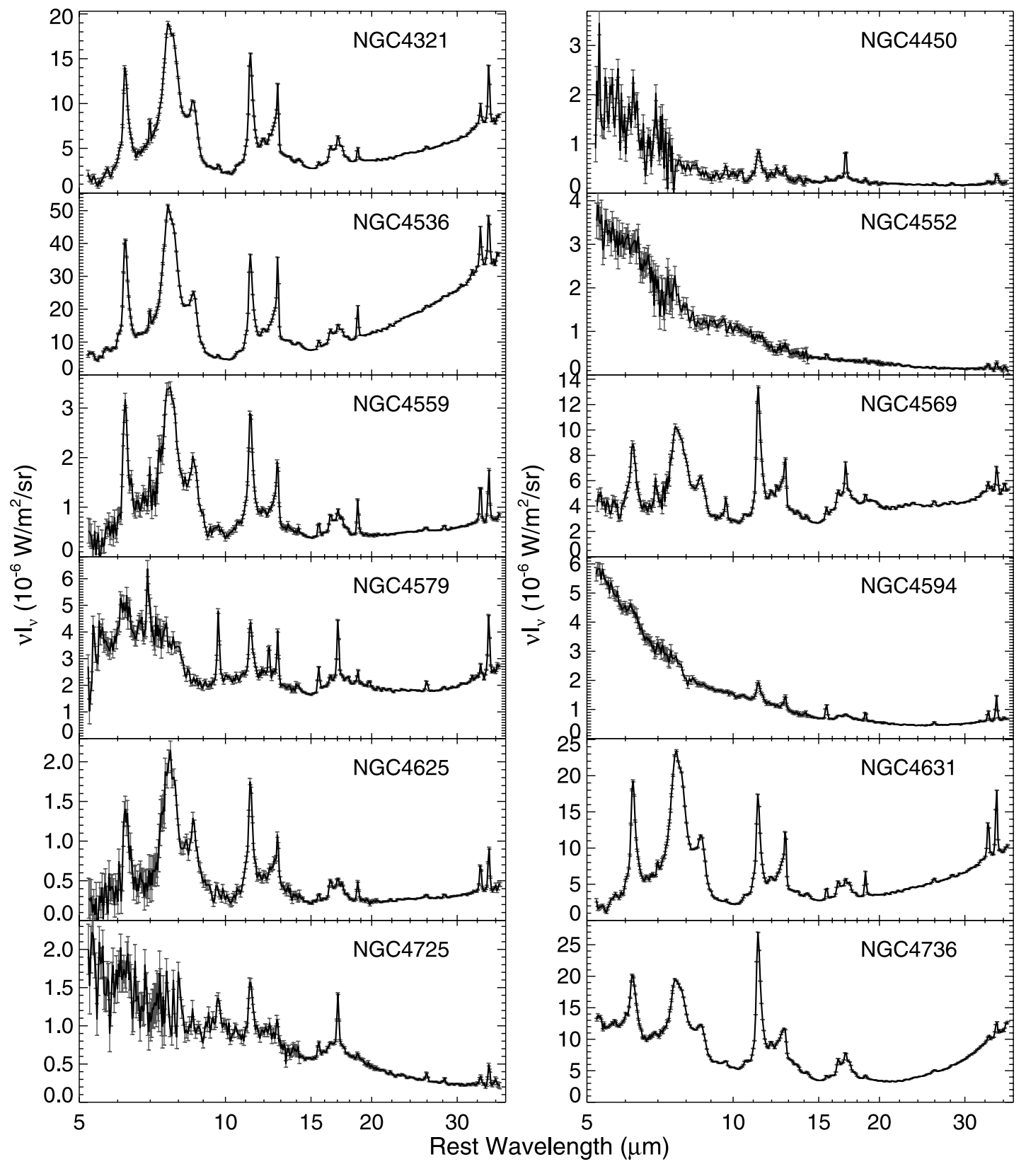

FIG. 1.-Continued

where $B_{\nu}$ is the blackbody function, $T_{\star}$ is the temperature of the stellar continuum, $T_{m}$ are the $n=M$ thermal dust continuum temperatures, the $I_{r}(\nu)$ are the $n=R$ resolved (dust) and unresolved (line) emission features, and $\tau_{\lambda}$ is the dust opacity. These features are described in detail below.

\subsubsection{Starlight}

The infrared emission from older stellar populations is represented by blackbody emission at fixed temperature $T_{\star}=5000 \mathrm{~K}$, similar to the temperature of the stars that dominate stellar emission redward of 3-5 $\mu \mathrm{m}$. This temperature was found to best represent the stellar continuum in average Starburst99 spectral energy distributions (SEDs; Leitherer et al. 1999) for stellar populations older than $100 \mathrm{Myr}$ and wavelength longward of $3 \mu \mathrm{m}$. For shorter rest-frame wavelengths, or in fits involving nearinfrared (NIR) photometry, including a more realistic suite of stellar SEDs would be appropriate, as would considering the very hot $\left(10^{3} \mathrm{~K}\right)$ small grain NIR dust emission component inferred in ISOPHOT spectra of normal galaxies (Lu et al. 2003). These components are unimportant beyond rest-frame wavelengths of $3-5 \mu \mathrm{m}$.

\subsubsection{Dust Continuum}

We allow up to $M=8$ thermal dust continuum components represented by modified blackbodies at fixed temperatures $T_{m}=$ $\{35,40,50,65,90,135,200,300 \mathrm{~K}\}$, with $\nu^{2}$ emissivity normalized at $\lambda_{0}=9.7 \mu \mathrm{m}$. Although the bulk of the dust in galaxies is heated to relatively low equilibrium temperatures $(\sim 15-20 \mathrm{~K}$; see B. T. Draine et al. 2007, in preparation), these grains are too cold to make any contribution at $\lambda<40 \mu \mathrm{m}$ and are not considered here. Contributions from a hot grain continuum, which would be important in strong AGN sources, are also not included; if present, e.g., for strong SINGS AGNs like NGC 1566 and NGC 5194, these continuum components would be absorbed by 


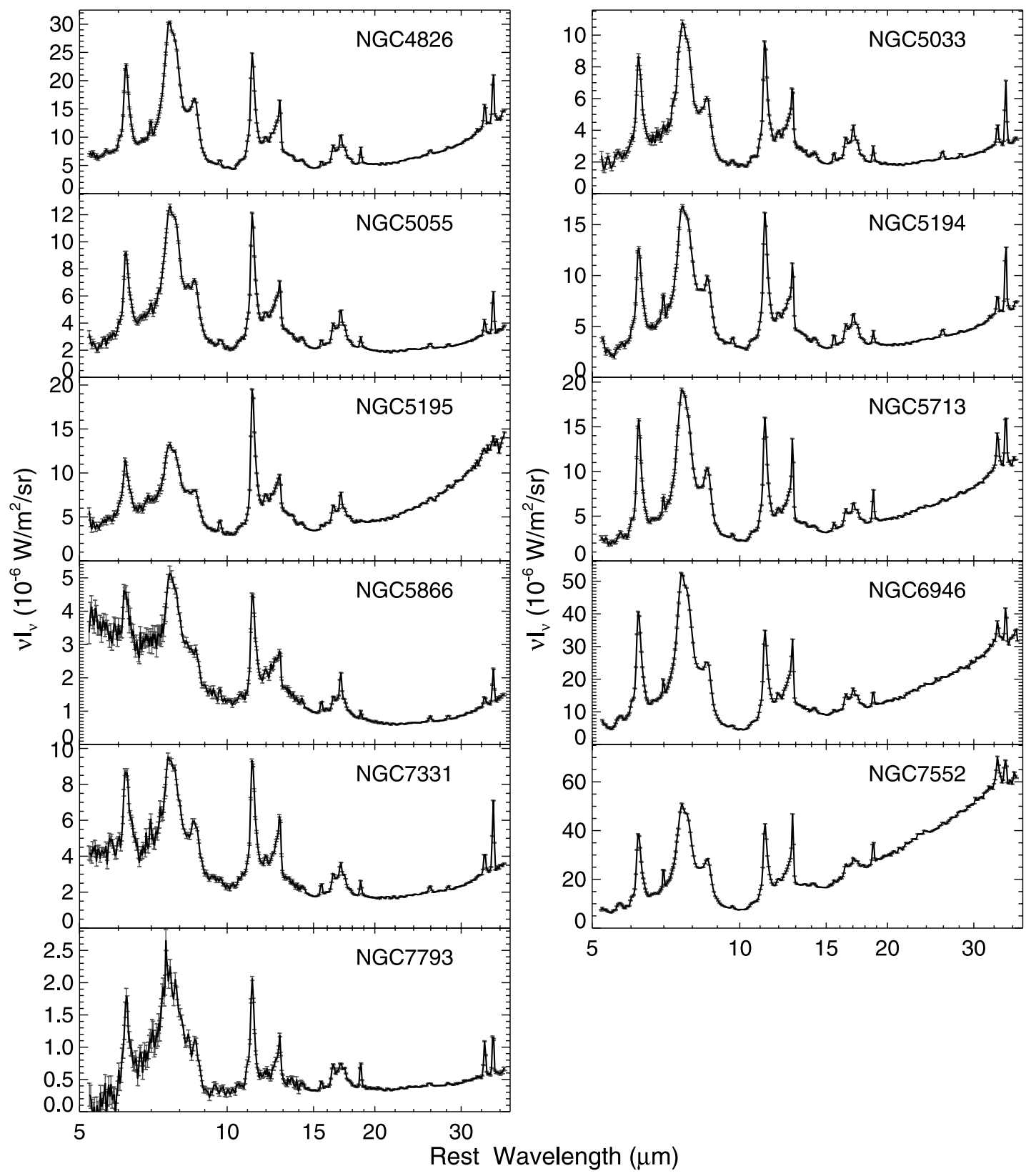

FIG. 1.-Continued

a combination of warm thermal and stellar continuum. The dust continuum is thus determined by the $M$ parameters $\tau_{m}$, each required to be nonnegative (but not nonzero). The number of components was chosen to reproduce smooth distributions of grain temperature, although reducing the number (but not temperature range) of these components would not significantly impact the resulting decomposition.

No special significance is attached to the strength of individual temperature components; rather, they are chosen to produce a physically realistic dust continuum underlying the discrete lines and dust features. Including 70 and $160 \mu \mathrm{m}$ MIPS photometry, along with IRAC and near-infrared photometry in matched apertures, would permit placing more significance on the resulting nonstochastic dust temperature distribution; this is not pursued here. For extracting PAH, silicate, and line strengths, such additional short- and long-wavelength constraints have lit- tle impact, and changes in the number and exact placement of the temperature bins also do not strongly affect the resulting feature parameters. On average, only five to six of the maximum allowed eight thermal dust components have nonnegligible power in a given model fit, although all components are well populated when considering the sample as a whole.

\subsubsection{Spectral Line Features}

A diverse collection of spectral lines is emitted in the MIR range probed by the IRS, including the $\mathrm{H}_{2} S(0)-\mathrm{H}_{2} S(6)$ pure rotational lines of molecular hydrogen and strong $\mathrm{H}$ II region and photodissociation region (PDR) cooling lines from low-ionization species such as $\mathrm{Si}^{+}$, with an ionization potential of $8.2 \mathrm{eV}$, to massive star and AGN tracers from $\mathrm{O}^{+3}(55 \mathrm{eV})$ and $\mathrm{Ne}^{+4}(97 \mathrm{eV})$. These lines are modeled primarily to allow realistic decomposition of blended features and the underlying continuum. The 
TABLE 2

Unresolved Line Parameters

\begin{tabular}{|c|c|c|}
\hline $\begin{array}{c}\text { Line } \\
\text { (1) }\end{array}$ & $\begin{array}{c}\lambda_{r} \\
(\mu \mathrm{m}) \\
(2)\end{array}$ & $\begin{array}{c}\text { FWHM } \\
(\mu \mathrm{m}) \\
(3)\end{array}$ \\
\hline $\mathrm{H}_{2} S(7) \ldots \ldots \ldots$ & 5.511 & 0.053 \\
\hline 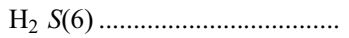 & 6.109 & 0.053 \\
\hline $\mathrm{H}_{2} S(5) \ldots \ldots$ & 6.909 & 0.053 \\
\hline$\ldots \ldots \ldots \ldots \ldots \ldots \ldots$ & 6.985 & 0.053 \\
\hline 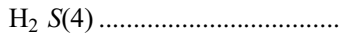 & 8.026 & 0.100 \\
\hline 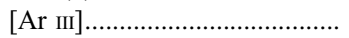 & 8.991 & 0.100 \\
\hline $\mathrm{H}_{2} S(3) \ldots \ldots$ & 9.665 & 0.100 \\
\hline [S IV] & 10.511 & 0.100 \\
\hline 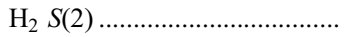 & 12.278 & 0.100 \\
\hline 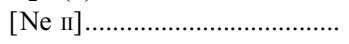 & 12.813 & 0.100 \\
\hline 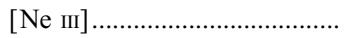 & 15.555 & 0.140 \\
\hline 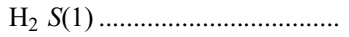 & 17.035 & 0.140 \\
\hline$[\mathrm{S}$ III] & 18.713 & 0.140 \\
\hline 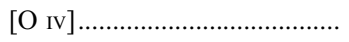 & 25.910 & 0.340 \\
\hline 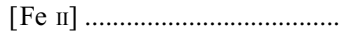 & 25.989 & 0.340 \\
\hline 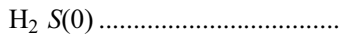 & 28.221 & 0.340 \\
\hline 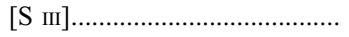 & 33.480 & 0.340 \\
\hline$[\mathrm{Si}$ II] & 34.815 & 0.340 \\
\hline
\end{tabular}

Notes.-Col. (1): Line name. Col. (2): Central wavelength. Col. (3): Full width at half-maximum.

strong line pair ratio $[\mathrm{Ne} \mathrm{III}] /[\mathrm{Ne} \mathrm{II}]$ provides an indicator of radiation hardness unaffected by all but the heaviest dust extinction (Thornley et al. 2000).

Spectral lines in the model are represented by Gaussian profiles, with widths set per instrument module based on direct measurements of unresolved features and shifted into the rest frame. The lines that are fitted to the spectra are listed in Table 2. Line wavelengths are allowed to vary by $0.05 \mu \mathrm{m}$ (roughly $\frac{1}{2}$ of a spectral pixel, approximately the wavelength calibration uncertainty at the blue ends of each IRS order), and line widths are allowed to vary by $10 \%$ from their initial default values. The central intensity of all lines is required to be nonnegative.

\subsubsection{Dust Features}

Dust features are represented by individual and blended Drude profiles:

$$
I_{\nu}^{(r)}=\frac{b_{r} \gamma_{r}^{2}}{\left(\lambda / \lambda_{r}-\lambda_{r} / \lambda\right)^{2}+\gamma_{r}^{2}},
$$

where $\lambda_{r}$ is the central wavelength of the feature, $\gamma_{r}$ is the fractional FWHM, and $b_{r}$ is the central intensity, which is required to be nonnegative. The Drude profile is the theoretical frequency profile for a classical damped harmonic oscillator and is a natural choice to model PAH emission features (and other emission and absorption bands, e.g., the $2175 \AA$ extinction feature; Fitzpatrick $\&$ Massa 1986). The Drude profile, like the Lorentzian, has more power in the extended wings than a Gaussian. Power in the overlapping wings of multiple bands can dominate the apparent continuum, especially in the $5-12 \mu \mathrm{m}$ region. The integrated intensity of the Drude profile is

$$
I^{(r)} \equiv \int I_{\nu}^{(r)} d \nu=\frac{\pi c}{2} \frac{b_{r} \gamma_{r}}{\lambda_{r}} .
$$

Table 3 lists all modeled dust features, some of which are represented by up to three subfeatures, grouped as blended com-
TABLE 3

\begin{tabular}{|c|c|c|}
\hline $\begin{array}{c}\lambda_{r} \\
(\mu \mathrm{m}) \\
(1)\end{array}$ & $\begin{array}{l}\gamma_{r} \\
(2)\end{array}$ & $\begin{array}{c}\text { FWHM } \\
(\mu \mathrm{m}) \\
(3)\end{array}$ \\
\hline $5.27 \ldots$ & 0.034 & 0.179 \\
\hline $5.70 \ldots \ldots \ldots \ldots \ldots . . . .$. & 0.035 & 0.200 \\
\hline $6.22 \ldots \ldots \ldots \ldots \ldots \ldots \ldots$ & 0.030 & 0.187 \\
\hline 6.69 & 0.070 & 0.468 \\
\hline $7.42^{\mathrm{a}}$ & 0.126 & 0.935 \\
\hline $7.60^{\mathrm{a}}$ & 0.044 & 0.334 \\
\hline $7.85^{\mathrm{a}} \ldots \ldots \ldots \ldots \ldots \ldots \ldots$ & 0.053 & 0.416 \\
\hline $8.33 \ldots \ldots .$. & 0.050 & 0.417 \\
\hline ........................ & 0.039 & 0.336 \\
\hline …................. & 0.020 & 0.214 \\
\hline 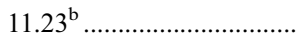 & 0.012 & 0.135 \\
\hline $11.33^{\mathrm{b}} \ldots \ldots$ & 0.032 & 0.363 \\
\hline $11.99 \ldots$ & 0.045 & 0.540 \\
\hline $12.62^{\mathrm{c}}$. & 0.042 & 0.530 \\
\hline $12.69^{\mathrm{c}}$ & 0.013 & 0.165 \\
\hline $13.48 \ldots \ldots \ldots \ldots$ & 0.040 & 0.539 \\
\hline $14.04 \ldots \ldots$ & 0.016 & 0.225 \\
\hline 14.19 & 0.025 & 0.355 \\
\hline 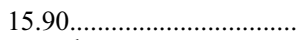 & 0.020 & 0.318 \\
\hline $16.45^{\mathrm{d}}$. & 0.014 & 0.230 \\
\hline $17.04^{\mathrm{d}}$. & 0.065 & 1.108 \\
\hline $17.375^{\mathrm{d}}$. & 0.012 & 0.209 \\
\hline $17.87^{\mathrm{d}} .$. & 0.016 & 0.286 \\
\hline $18.92 \ldots \ldots$ & 0.019 & 0.359 \\
\hline 33.10 & 0.050 & 1.655 \\
\hline
\end{tabular}

Dust Feature Parameters

Notes.-Col. (1): Central wavelength. Col. (2): Fractional FWHM. Col. (3): Full width at half-maximum.

a $7.7 \mu \mathrm{m}$ complex.

b $11.3 \mu \mathrm{m}$ complex.

c $12.7 \mu \mathrm{m}$ complex.

d $17 \mu \mathrm{m}$ complex.

plexes. The $17 \mu \mathrm{m}$ complex has two resolvable components, at $16.4 \mu \mathrm{m}$ (Moutou et al. 2000) and $17.4 \mu \mathrm{m}$ (Smith et al. 2004; Werner et al. 2004; see Fig. 4 below). For the other blends (7.7, 11.3 , and $12.7 \mu \mathrm{m})$, subfeatures were adopted to best reproduce the band shape. Unresolved complexes of this type are considered only in aggregate, and the subfeatures comprising them are not intended to represent distinct physical emission bands. The number and placement of these subfeatures were determined by training the model on a subset of 25 high-signal galaxies from the sample, by fixing all other fitting components, and allowing the width and central wavelengths to vary. A similar optimization was performed for the weaker individual features. Once determined, feature positions and widths were held fixed. Related fitting approaches have been used with ISO spectra (Boulanger et al. 1998; Verstraete et al. 2001).

\subsubsection{Features from 5 to $10 \mu \mathrm{m}$}

The bulk of the PAH emission between 5 and $12 \mu \mathrm{m}$ is comprised of the well-studied set of features discovered in planetary nebulae by Gillett et al. (1973) and probed in detail in Galactic objects with ISO (e.g., reflection nebula NGC 7023; Cesarsky et al. 1996). The weak $8.33 \mu \mathrm{m}$ band, which sits between the prominent 7.7 and $8.6 \mu \mathrm{m}$ features, was tentatively detected in the ISO SWS spectra of M82 (Sturm et al. 2000) and is present in many of our spectra, serving in most cases only to fill in the saddle between the two stronger features. A weak new feature in external galaxies occurs at $6.69 \mu \mathrm{m}$ (see also Werner et al. 2004). 


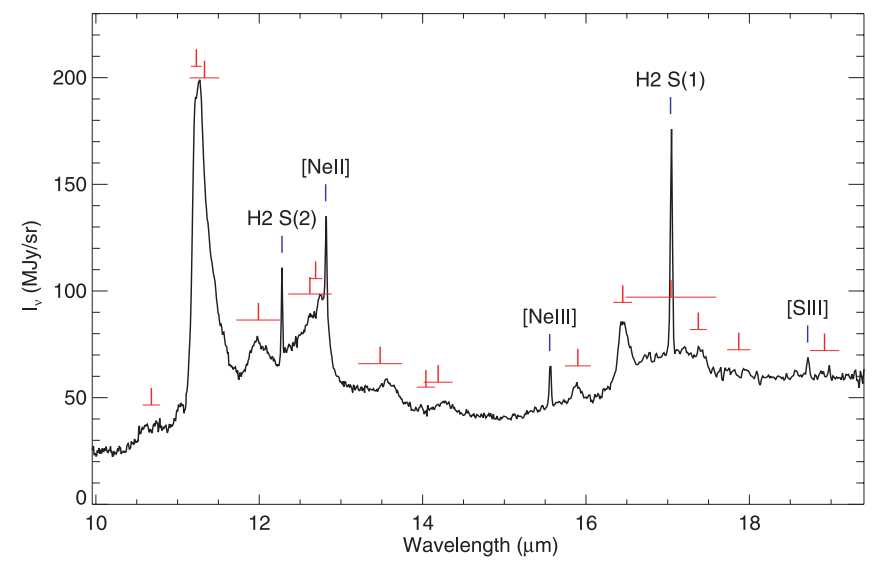

FIG. 2.-High-resolution spectrum of NGC 5195 (M51b) illustrating the subset of features from 10 to $19 \mu \mathrm{m}$ identified in the full low-resolution sample. The PAH feature central wavelength and FWHM for features from Table 3 with central wavelengths $>10 \mu \mathrm{m}$ are indicated above the spectra, and unresolved emission lines in the spectrum are also labeled.

A small number of stellar-dominated spectra from elliptical and other early-type systems in the sample show broad $10 \mu \mathrm{m}$ excess (e.g., NGC 4552), which has been attributed in similar systems to silicate emission from the winds of asymptotic giant branch populations (Bressan et al. 2006). These features are not considered in our fit and are weak enough not to influence the recovery of stellar continuum in these cases, although they can induce spurious dust features (especially at $7.7 \mu \mathrm{m}$ ) and for this reason are excluded from results pertaining to these features.

\subsubsection{Features from 10 to $20 \mu \mathrm{m}$}

Along with the strong bands at $11.3,12.7$, and $17.0 \mu \mathrm{m}$, a variety of weak features are seen in most low-resolution spectra between 10 and $20 \mu \mathrm{m}$. The $10.7 \mu \mathrm{m}$ band, which falls in the (potentially silicate extincted) trough between the main 7.7 and $11.3 \mu \mathrm{m}$ features, was tentatively identified in the ISO spectra of M82 (Sturm et al. 2000) and is not uncommon in the sample, occasionally blending with nearby [S IV] at $10.51 \mu \mathrm{m}$. Other weak bands are found at 12.0, 12.6, 13.6, 14.3, 15.9, 16.4, and $17.4 \mu \mathrm{m}$. Sturm et al. (2000) identified all of these features in ISO SWS spectra of the bright starburst galaxies NGC 253 and M82, and many of the individual subfeatures were first discovered in Galactic sources (e.g., Moutou et al. 2000). The $14.3 \mu \mathrm{m}$ dust feature may be blended with [Ne v] $14.32 \mu \mathrm{m}$ in sources with AGN nuclei. Another very weak feature at $18.92 \mu \mathrm{m}$ is likely the same as the $19 \mu \mathrm{m}$ feature found in Spitzer spectra of reflection nebula NGC 7023 by Werner et al. (2004). It is blended with [S III] at $18.7 \mu \mathrm{m}$. Dust features $>10 \mu \mathrm{m}$ are marked in Figure 2 on the high-resolution IRS nuclear spectrum of NGC 5195 (M51b). Note in particular that the $12.7 \mu \mathrm{m}$ PAH band, which is blended with [Ne II] at low resolution, cleanly separates with $[\mathrm{Ne}$ II $]$ to the red wing of the blend at higher resolution.

Interestingly, the strongest feature at $\lambda>12 \mu \mathrm{m}$, the broad band centered at $17.04 \mu \mathrm{m}$, was not seen in the $I S O$ spectra of the brightest starbursts and was first identified in an extragalactic source in the SINGS galaxy NGC 7331 (Smith et al. 2004). Despite the strength of the $17 \mu \mathrm{m}$ complex, which can carry power comparable to the prominent $11.3 \mu \mathrm{m}$ band (see $\S 5.4$ ), its large width $(\sim 1.1 \mu \mathrm{m})$ and the lack of available low-resolution spectroscopy at wavelengths above $16 \mu \mathrm{m}$ combined to make this prominent band difficult to detect with $I S O$. The $17 \mu \mathrm{m}$ feature was reported simultaneously in Spitzer spectra of reflection nebula NGC 7023 (Werner et al. 2004). Evidence for a similar

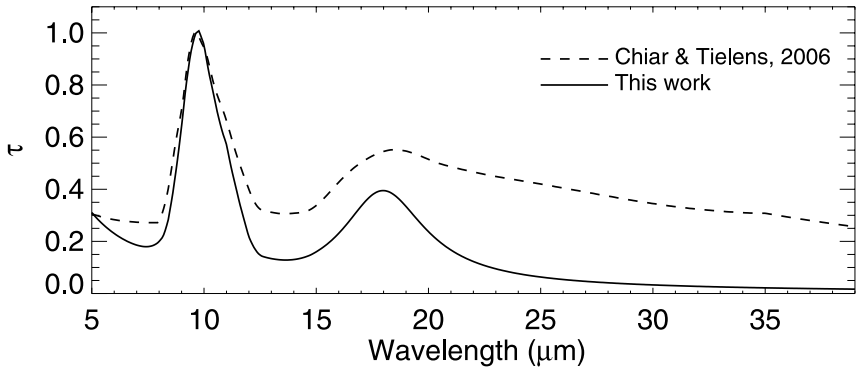

FIG. 3.-Adopted extinction curve, along with the curve of Chiar \& Tielens (2006).

band complex was found in ISO SWS spectra of Galactic sources (Beintema et al. 1996; Van Kerckhoven et al. 2000).

\subsubsection{Features beyond $20 \mu \mathrm{m}$}

A single feature was included beyond $20 \mu \mathrm{m}$ : a broad band centered at $33.1 \mu \mathrm{m}$. Although not present in all sources, it underlies the [Si II] and [S III] lines and is required to obtain accurate line fluxes for these lines. Sturm et al. (2000) notice a broad plateau from 33 to $34 \mu \mathrm{m}$ in three ISO SWS galaxy spectra and attribute it to crystalline silicates. A similar plateau was noted in the SWS spectra of planetary nebulae (Waters et al. 1998). This feature is strong in $\sim \frac{1}{6}$ of our sample (see, e.g., NGC 4321), with no correlation of the feature's strength with silicate extinction (see $\S 5.2)$.

\subsubsection{Dust Extinction}

We allow for absorption by dust with extinction properties similar to Milky Way dust. We take the infrared extinction to consist of a power law plus silicate features peaking at 9.7 and $18 \mu \mathrm{m}$ :

$$
\tau(\lambda)=\tau_{9.7}\left[(1-\beta) P_{\mathrm{Si}}(\lambda)+\beta(9.7 / \lambda)^{1.7}\right]
$$

where $\tau_{9.7}$ is the total extinction at $9.7 \mu \mathrm{m}$ and $P_{\mathrm{Si}}(\lambda)$ is the normalized silicate profile function, with $P_{\mathrm{Si}}(9.7 \mu \mathrm{m})=1$. We adopt $\beta=0.1$; with this choice, $\tau(3.6 \mu \mathrm{m})=0.54 \tau(9.7 \mu \mathrm{m})$, which is within the current uncertainty range regarding extinction from 3 to $8 \mu \mathrm{m}$ (see, e.g., Draine \& Li 2001). The $9.7 \mu \mathrm{m}$ component of $P_{\mathrm{Si}}(\lambda)$ is taken from the Galactic center profile of Kemper et al. (2004) between 8.0 and $12.5 \mu \mathrm{m}$, with $P_{\mathrm{Si}}(8 \mu \mathrm{m})=$ 0.06 . For $\lambda<8 \mu \mathrm{m}$, we smoothly extend this profile by assuming $P_{\mathrm{Si}}(\lambda)=P_{\mathrm{Si}}(8 \mu \mathrm{m}) e^{2.03(\lambda-8 \mu \mathrm{m})}$. For $\lambda>12.7 \mu \mathrm{m}$, we add a Drude profile extinction component peaking at $18 \mu \mathrm{m}$, with $\mathrm{FWHM}=4.45 \mu \mathrm{m}\left(\gamma_{r}=0.247\right)$, smoothly joining the two profiles between 12.5 and $12.7 \mu \mathrm{m}$. The fitted silicate depth $\tau_{9.7}$ is required to be nonnegative; no silicate emission was included in the model. We also explored using the Galactic center extinction curve of Chiar \& Tielens (2006) with similar values of $\tau_{9.7}$ derived, but the much larger extinction at long wavelengths $\left(A_{30 \mu \mathrm{m}} \sim A_{8 \mu \mathrm{m}}\right)$ produced poorer overall fits in absorbed galaxies with our choice of dust continuum temperatures. The adopted extinction curve is shown in Figure 3.

The default extinction geometry is taken to be absorbing dust fully mixed with emitting stars and grains; a uniform foreground screen of dust is also considered. Since all components of the model are presumed to be equally affected by dust extinction, line and feature strengths are automatically corrected to their extinction-free values. This implicit correction may be unphysical if the material emitting the MIR continuum, from which the dust absorption is primarily determined, and the PAH-emitting 


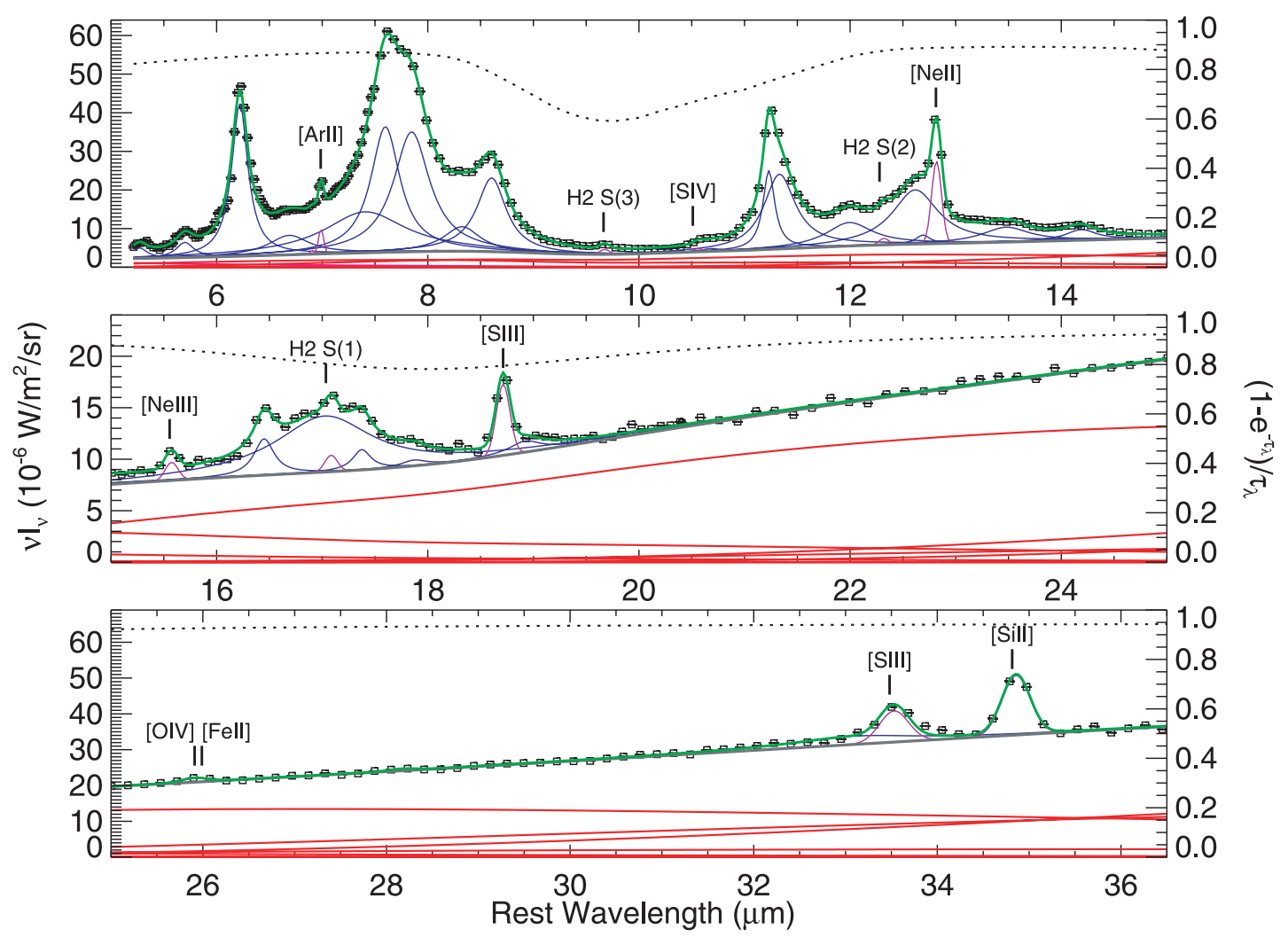

FIG. 4.-Detailed decomposition of NGC 1482 from 5 to $37 \mu \mathrm{m}$. Red solid lines represent the thermal dust continuum components, the magenta line shows the stellar continuum (weak in this case), and the thick gray line shows the total (dust+stellar) continuum. Blue lines set above the total continuum are dust features, while the narrower violet peaks are unresolved atomic and molecular spectral lines. All components are diminished by the fully mixed extinction, indicated by the dotted black line, with axis at right. The solid green line is the full fitted model, plotted on the observed flux intensities and uncertainties.

material undergo differential extinction. The TIR luminosity estimates described in $\S 3$ are not extinction corrected, but the correction for $\lambda>25 \mu \mathrm{m}$, where the bulk of the TIR power emerges, is negligible.

\subsection{Fitting and Uncertainties}

The model in equation (1) is fitted to the observed flux intensity by minimizing the global $\chi^{2}$ using the Levenberg-Marquardt algorithm, modified to allow the values of individual fit parameters to be fixed within allowed limits. Formal statistical uncertainties, derived from the IRS pipeline ramp fitting uncertainties, are returned for each fit parameter, and full uncertainties in combined quantities such as integrated intensities are formulated using the full covariance matrix. In particular, for estimating uncertainties in the integrated intensity of blended dust features, correlations between components can introduce significant cross term corrections to the propagation of error. For upper limits, additional uncertainty estimates, based on the residuals of the observed flux intensity from the best-fitting model, are adopted. The response of the model to a variety of simulated changes to the continuum was tested, and the strengths of dust features recovered were found to be robust against such variations. In particular, adding strong stellar continuum to $\mathrm{PAH}$-dominated spectra and refitting resulted in feature fluxes that changed by less than $2 \%$.

\subsection{Example Decomposition and Tool Availability}

The detailed components of the spectral decomposition method are illustrated in Figure 4 for NGC 1482 and in Figure 5 for four additional examples: two sources with significant dust extinction and two sources with starlight dominating their short-wavelength continuum. All galaxies in the sample are well reproduced by the modeled components. A tool implementing the decomposition method is available in PAHFIT, an IDL package developed for the decomposition of low-resolution IRS spectra comprised of stellar and thermal dust continuum (not including very hot dust in strong AGNs), dust features, line emission, and silicate absorption. Source code and documentation for PAHFIT are available. ${ }^{14}$

\section{RESULTS}

The spectral decomposition of all 59 sample galaxies was used to investigate silicate extinction, the energetics of $\mathrm{PAH}$ emission, variations in the large number of individual and blended PAH features recovered, and the impact of these variations on studies of high-redshift galaxies in deep broadband surveys. Recovered strengths for all important dust features and the [ $\mathrm{Ne}$ II] and [ $\mathrm{Ne}$ III] lines are given in Table 4.

\subsection{The Nature of the $17 \mu \mathrm{m}$ Emission Band}

The $17 \mu \mathrm{m}$ PAH complex is the strongest band longward of $12 \mu \mathrm{m}$. In contrast to the highly variable plateau reported by Peeters et al. (2004), all SINGS spectra exhibit a dominant component of the $17 \mu \mathrm{m}$ complex that varies very little in position and width. This uniformity could be a result of beam averaging over a range of physical conditions in PDRs drawn from similar environmental distributions, or perhaps an indication that the Galactic sources considered by Peeters et al. (2004) were dominated by atypical band carriers or radiation environments. The distinct flanking subfeatures at 16.4 and $17.4 \mu \mathrm{m}$ contribute

14 See http://sings.stsci.edu/pahfit. 


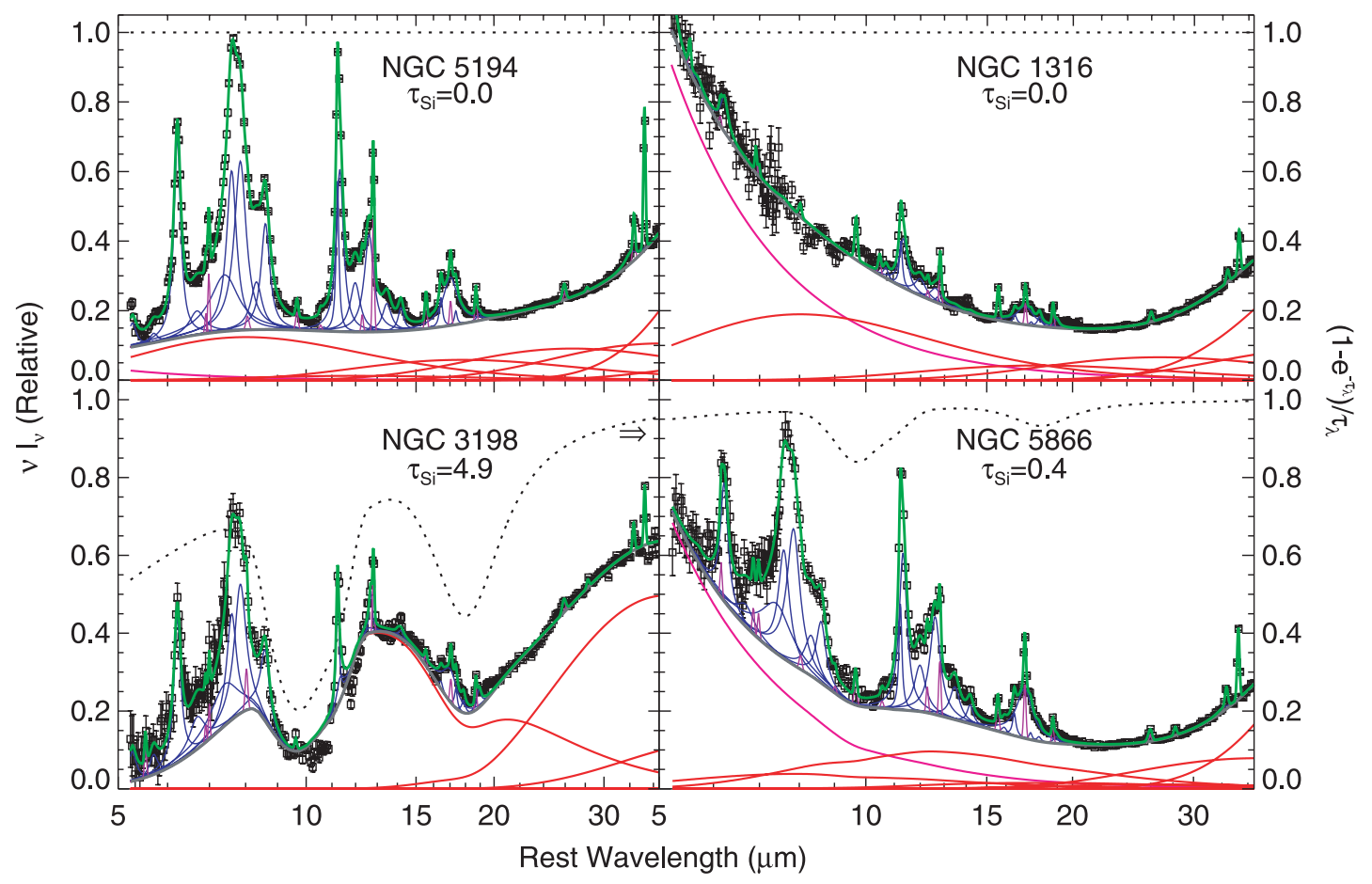

FIG. 5.-Example decomposition of four SINGS spectra, for sources with and without significant silicate extinction, and dominated by either stellar or thermal dust continuum at short wavelengths. All symbols are as in Fig. 4. Logarithmic wavelength axis.

only $\sim 20 \%$ of the total power in the complex, with the remainder contained in the broad (FWHM $\sim 1 \mu \mathrm{m}$ ) feature centered at $17.04 \mu \mathrm{m}$. A small number of galaxy spectra in the sample do exhibit an apparent plateau-like extension of the $17 \mu \mathrm{m}$ band to $\sim 19 \mu$ m (e.g., NGC 1566, NGC 4569), but it does not correlate with the strength of the $17 \mu \mathrm{m}$ feature or any other PAH band and may arise from a different carrier.

Although laboratory spectra of PAH mixtures contain many features between 15 and $20 \mu \mathrm{m}$ presumed due to $\mathrm{C}-\mathrm{C}-\mathrm{C}$ outof-plane bending modes (Peeters et al. 2004), it is of interest to compare the general behavior of the $17 \mu \mathrm{m}$ band with the other more traditional PAH bands. Figure 6 compares the integrated feature power of the 17 and $11.3 \mu \mathrm{m}$ complexes. The features correlate very well with each other over 2 orders of magnitude, strongly supporting the association of the $17 \mu \mathrm{m}$ complex with PAH emission. Some variations in the $17 \mu \mathrm{m} / 11 \mu \mathrm{m}$ intensity ratio are evident; these are explored in $\S 5.6$.

\subsection{The Incidence of Silicate Extinction}

Helou et al. (2000) found little evidence for silicate absorption in the 5.9-11.7 $\mu \mathrm{m}$ spectra of the ISO Key Project sample of 45 normal galaxies, and Sturm et al. (2000) also concluded that high-resolution $I S O$ spectra of bright starbursts like M82 were consistent with little or no silicates. With a more varied sample and access to the full $9.7 \mu \mathrm{m}$ and associated $18 \mu \mathrm{m}$ features, the SINGS spectra are uniquely suited to the investigation of moderate silicate absorption in a range of galaxy types.

Attempting to measure weak to moderate silicate absorption in spectra with strong PAH emission features can lead to ambiguous measures of both the feature strengths and the silicate optical depth. In systems dominated by deeply embedded infrared sources (e.g., many ULIRGs) with strong silicate absorption, strong to weak or even absent PAH bands, and accompanying shorter wavelength ice absorption features, the identification and measurement of the silicate and other absorption optical depths are straightforward (e.g., Armus et al. 2004; Spoon et al. 2006; Roussel et al. 2006). However, as the silicate optical depth decreases and the PAH strength relative to the local continuum increases, the PAH bands at $7.7 \mu \mathrm{m} / 8.6 \mu \mathrm{m}$ and $11.3 \mu \mathrm{m}$, which closely flank the main silicate absorption trough centered at $9.7 \mu \mathrm{m}$, can mask or mimic the true silicate absorption. With this absorption feature alone it is difficult to discriminate between moderate PAH emission superposed on a silicate-absorbed continuum and strong PAH features with a relatively weak underlying continuum. However, since the dust continuum at $15-20 \mu \mathrm{m}$ is fairly regular in the spectra of star-forming galaxies, increasing silicate absorption in the associated $18 \mu \mathrm{m}$ band results in a measurable change in the shape of the 15-20 $\mu \mathrm{m}$ continuum.

TABLE 4

Recovered Dust Feature and Line Strengths

\begin{tabular}{|c|c|c|c|c|c|c|c|c|c|c|c|}
\hline Galaxy & $6.2 \mu \mathrm{m}$ & $7.7 \mu \mathrm{m}$ & $8.3 \mu \mathrm{m}$ & $8.6 \mu \mathrm{m}$ & $11.3 \mu \mathrm{m}$ & $12.0 \mu \mathrm{m}$ & $12.6 \mu \mathrm{m}$ & $13.6 \mu \mathrm{m}$ & $16.4 \mu \mathrm{m}$ & $17 \mu \mathrm{m}$ & $17.4 \mu \mathrm{m}$ \\
\hline IC $4710 \ldots \ldots \ldots \ldots$ & $0.00 \mathrm{E}-00$ & $4.93 \mathrm{E}-08$ & $1.77 \mathrm{E}-09$ & $2.01 \mathrm{E}-08$ & $1.63 \mathrm{E}-08$ & $1.30 \mathrm{E}-08$ & $6.11 \mathrm{E}-09$ & $3.41 \mathrm{E}-09$ & $6.54 \mathrm{E}-10$ & $1.18 \mathrm{E}-09$ & $5.26 \mathrm{E}-10$ \\
\hline NGC $0024 \ldots$ & $2.26 \mathrm{E}-08$ & $6.40 \mathrm{E}-08$ & $6.23 \mathrm{E}-10$ & $1.39 \mathrm{E}-08$ & $2.12 \mathrm{E}-08$ & $4.48 \mathrm{E}-09$ & $8.58 \mathrm{E}-09$ & $2.06 \mathrm{E}-09$ & $2.54 \mathrm{E}-09$ & $1.42 \mathrm{E}-08$ & $9.43 \mathrm{E}-10$ \\
\hline NGC $0337 \ldots \ldots \ldots . . .$. & $1.70 \mathrm{E}-07$ & $4.97 \mathrm{E}-07$ & $3.29 \mathrm{E}-08$ & $1.00 \mathrm{E}-07$ & $1.38 \mathrm{E}-07$ & $3.60 \mathrm{E}-08$ & $7.88 \mathrm{E}-08$ & $2.10 \mathrm{E}-08$ & $9.94 \mathrm{E}-09$ & $5.85 \mathrm{E}-08$ & $3.18 \mathrm{E}-09$ \\
\hline
\end{tabular}

Noтеs.-Table 4 is published in its entirety in the electronic edition of the Astrophysical Journal. A portion is shown here for guidance regarding its form and content. The full data set contains $[\mathrm{Ne}$ II] and $[\mathrm{Ne}$ III] line intensities, as well as uncertainties for all values. 


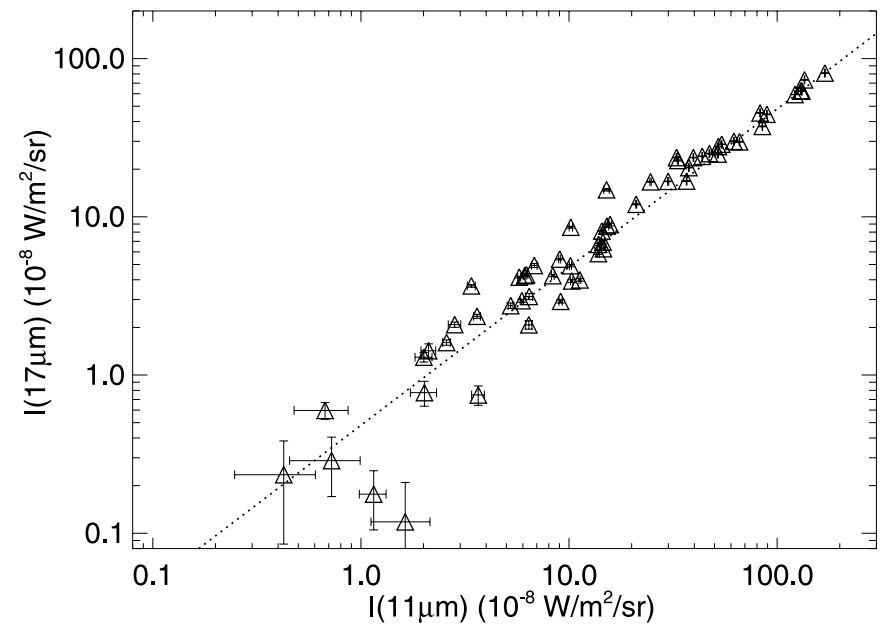

FIG. 6.-Correlation between the intensity of the 11.3 and $17 \mu \mathrm{m}$ PAH complexes, shown with a slope unity line.

A compelling example of this degeneracy in recovering silicate extinction from limited wavelengths around the $10 \mu \mathrm{m}$ band is found in NGC 5866. Lu et al. (2003) noted an unusually high value of $L(6.2 \mu \mathrm{m}) / L(7.7 \mu \mathrm{m})$ for this compact, edge-on disk galaxy. With access to the full 5-38 $\mu \mathrm{m}$ spectrum and identification of the nonnegligible silicate extinction $\tau_{9.7}=0.36$, the band ratio $L(6.2 \mu \mathrm{m}) / L(7.7 \mu \mathrm{m})$ is placed on the low end of the distribution (see Fig. 13 below). In this case, the strong photospheric continuum that dominates NGC 5866's spectrum out to $10 \mu \mathrm{m}$ may have further complicated accurate measurement of the band strengths.

Only $13.6 \%$, or 8 of the 59 sample spectra shown in Figure 1, require nonnegligible absorption by silicate dust to reproduce their spectra. These objects and their inferred silicate optical depths $\tau_{9.7}$ are listed in Table 5 , for the default fully mixed dust geometry, as well as a uniform foreground screen. The silicate depths are lower for the screen geometry, by roughly a factor of 2 . The mixed and screen models provide equally acceptable fits. Also given is $\chi^{2}\left(\tau_{\mathrm{Si}}=0\right) / \chi^{2}\left(\tau_{\mathrm{Si}}\right)$, the ratio of $\chi^{2}\left(\tau_{\mathrm{Si}}=0\right)$, formed by explicitly forcing the silicate depth to be zero, to the normal $\chi^{2}\left(\tau_{\mathrm{Si}}\right)$ including absorption. This ratio demonstrates the degree to which the fit is improved by adding silicate extinction and indicates that the decomposition method can robustly recover modest extinction with mixed silicate optical depths $\tau_{\mathrm{Si}} \gtrsim 0.35$, or screen depths half of that value. Thus, it appears that most normal galaxies do not exhibit dust extinction to the MIR-emitting material in their central few square kiloparsecs above $A_{V} \sim 5$ for fully mixed dust geometries, or $A_{V} \sim 3$ for foreground screens of dust (assuming $A_{V} / \tau_{\mathrm{Si}}=16.6$; Rieke \& Lebofsky 1985). Screen extinctions derived separately from optical nuclear spectra for the SINGS sample show $\left\langle A_{V}\right\rangle \sim$ 1.3, consistent with the inferred silicate depths (Dale et al. 2006). Brandl et al. (2006) found a larger range and greater incidence of silicate absorption depths in the nuclear spectra of a sample of starburst galaxies.

\subsection{Recovering PAH Strengths}

A widely used method for extracting PAH strength involves selecting narrow continuum regions free from line or feature emission, forming linear or cubic spline fits through these continuum points, and integrating the spectrum above this pseudocontinuum (e.g., Uchida et al. 2000; Peeters et al. 2002; Brandl et al. 2006). An application of this method to the SINGS spectrum of NGC 2798 is shown in Figure 7. The method's advan-
TABLE 5

Silicate Dust Extinction Sources

\begin{tabular}{|c|c|c|c|}
\hline \multirow[b]{2}{*}{ GaLAXY } & \multicolumn{2}{|c|}{$\tau_{9.7 \mu \mathrm{m}}$} & \multirow[b]{2}{*}{$\chi^{2}\left(\tau_{\mathrm{Si}}=0\right) / \chi^{2}\left(\tau_{\mathrm{Si}}\right)$} \\
\hline & Mixed & Screen & \\
\hline NGC 1266............................... & 2.05 & 0.91 & 1.868 \\
\hline 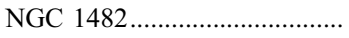 & 1.04 & 0.37 & 1.346 \\
\hline 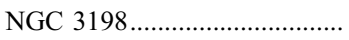 & 4.88 & 1.90 & 4.978 \\
\hline NGC 4536............................... & 0.37 & 0.18 & 1.086 \\
\hline 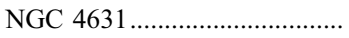 & 0.40 & 0.20 & 1.031 \\
\hline 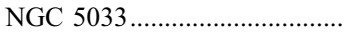 & 0.41 & 0.20 & 1.058 \\
\hline 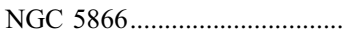 & 0.36 & 0.17 & 1.060 \\
\hline 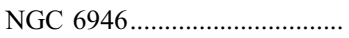 & 0.76 & 0.37 & 1.258 \\
\hline
\end{tabular}

tage is that it is relatively straightforward to apply and requires no specific assumptions regarding the nature of the continuum. The disadvantage is that locating nearby continuum anchors free from line, feature, or absorption contamination can prove difficult and to some degree arbitrary, with the resulting feature strengths depending sensitively on the exact choice of fiducial continuum points. This problem is compounded in low-quality spectra, where continuum regions free from these contaminating effects may be less readily identified.

To compare the band strengths recovered by the spline fitting and full decomposition techniques, we have applied the cubic spline method for the features at $6.2,7.7,8.6$, and $11.3 \mu \mathrm{m}$ to the full sample, fixing the rest wavelength continuum pivots following Peeters et al. (2002). Figure 7 illustrates the chosen fixed rest-frame continuum anchor points: three points for $6.2 \mu \mathrm{m}$, four for $11.3 \mu \mathrm{m}$, and five for the $7.7+8.6 \mu \mathrm{m}$ blend. The PAH bands at 12.6 and $17 \mu \mathrm{m}$ are blended with strong emission lines ([Ne II] and $\mathrm{H}_{2} S(1)$ ) and for this reason cannot be recovered with spline-based methods.

Figure 8 shows the PAH strengths recovered from full feature decomposition versus spline fitting as a function of the feature equivalent width [defined as $\int\left(I_{\nu}-I_{\nu}^{\text {cont }}\right) / I_{\nu}^{\text {cont }} d \lambda$ ]. Equivalent widths of the shortest wavelength features can be undefined, since the underlying continuum can vanish. In these cases, the profile-weighted average continuum was substituted. For the two unblended features in clean continuum regions, at 6.2 and $11.3 \mu \mathrm{m}$, the decomposition and spline-based methods yield feature powers differing by an approximately constant ratio, $L($ decomp $) / L($ spline $) \sim 1.75$, independent of the equivalent width of the feature. Since a fraction $f_{\text {wing }} \sim 59 \%$ of the power in a Drude profile lies outside its FWHM, it is not surprising that the offset between the two is roughly $1 / f_{\text {wing }}$.

For the blended features at 7.7 and $8.6 \mu \mathrm{m}$, however, the fraction of the full feature strength recovered by the spline method is lower, with larger scatter. This results from the continuum anchor placement in the filled trough between these features, located to ensure that the deblended peaks are independent. The overall scatter is probably somewhat larger than would have been achieved with hand-tuned spline continuum placement in all spectra. However, the large systematic offset would remain, such that the spline-based methods underestimate by a factor of 3-6 the full 7.7 and $8.6 \mu \mathrm{m}$ feature strengths. Table 6 lists the average offsets for feature equivalent widths larger than $0.1 \mu \mathrm{m}$, after applying an iterative $3 \sigma$ trimming of outliers. These $L($ decomp $) / L$ (spline) factors can be used to scale spline-recovered PAH fluxes to estimate crudely the total power in the PAH features, although, if possible, a more accurate estimate will be obtained directly using a decomposition method like the one described in $\S 4$. They may have dependence on the form of 


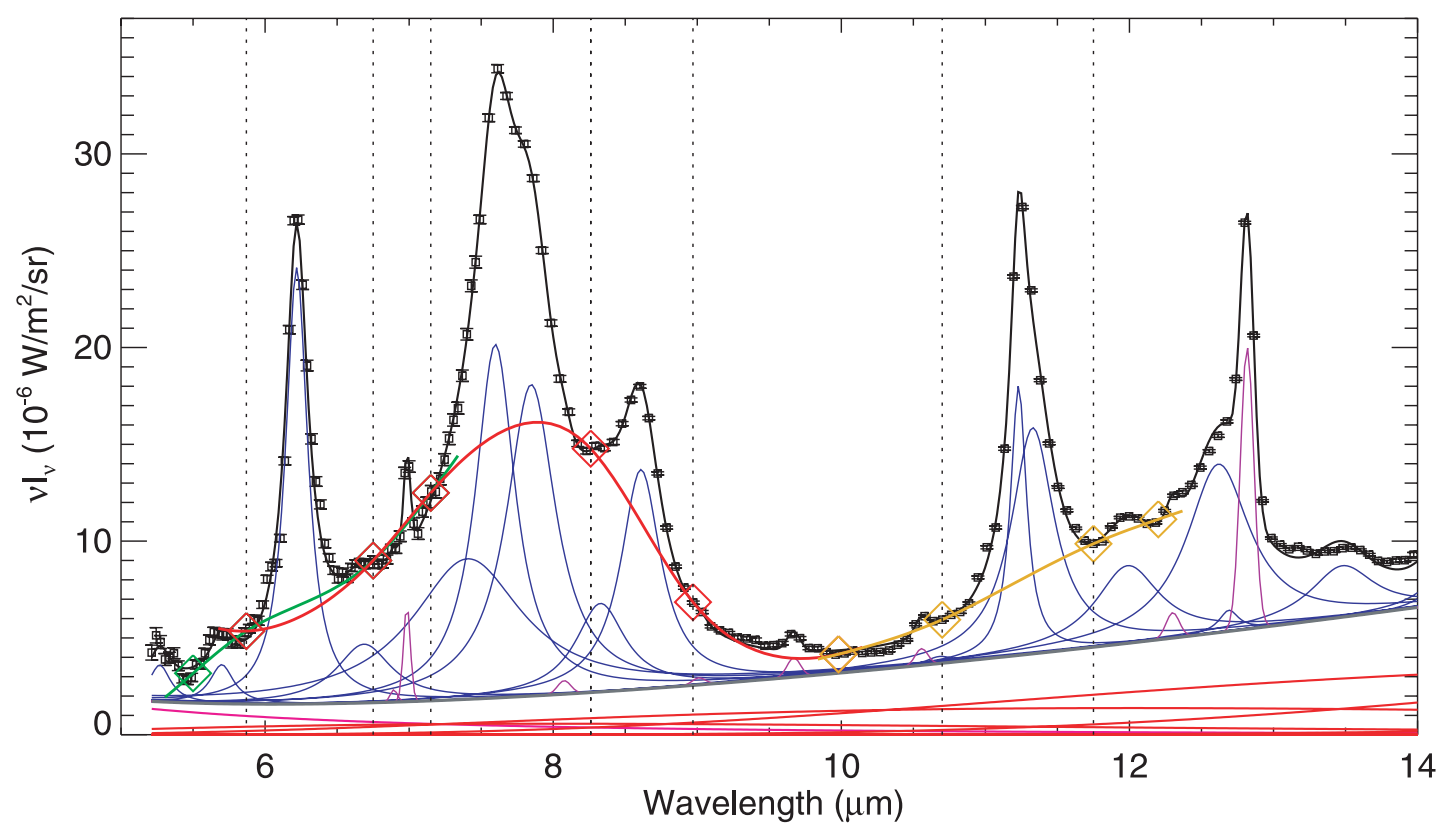

FIG. 7.-Example comparing the spline-based and full feature+continuum decomposition in SINGS galaxy NGC 2798. Three different spline fits are shown with continuum anchor points: in green (leftmost), underlying the $6.2 \mu \mathrm{m}$ feature, in red (center), underlying both the 7.7 and $8.6 \mu \mathrm{m}$ features, and in orange (rightmost), under the $11.3 \mu \mathrm{m}$ feature. Vertical dotted lines mark the ranges over which the spline-subtracted features are integrated. The fitted continuum, dust, and line features are as in Fig. 4.

underlying continuum and presence of silicate or other absorption and thus may not be appropriate for all spectra.

\subsection{PAH Energetics}

The total power emitted in the PAH bands can be large, and the current sample provides an opportunity to form a census of the energetic importance of individual features, independent of the underlying MIR continuum, which can be dominated by widely differing emission processes and environments and depend on the details of the stellar and dust grain populations. In Figure 9 the fractional power relative to the total PAH luminosity is shown for all significant features present in the sample.

The $7.7 \mu \mathrm{m}$ PAH complex can contribute nearly one-half of the total PAH luminosity and deliver up to $10 \%$ of the total infrared luminosity alone. In aggregate, the combined power output of all PAH features ranges from a few percent up to

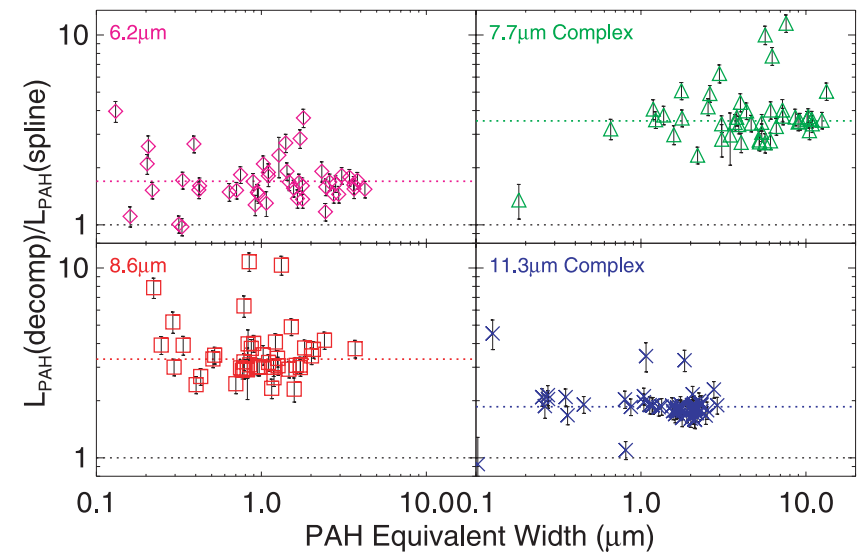

FIG. 8.- Ratio between the PAH feature strength from the full spectral decomposition and the PAH strength recovered from spline-based continuum fitting vs. the equivalent width measured from the decomposition. Mean and unity values are indicated with horizontal dotted lines.
$20 \%$ of the total infrared. Figure 10 shows the distribution of $L_{\mathrm{PAH}} / L_{\mathrm{TIR}}$ in the apertures considered, for the full sample, and separately for galaxies with evidence of nuclear Seyfert or LINER emission in their nuclear optical spectra and galaxies with only $\mathrm{H}$ II region or starburst classifications. The peak is near $L_{\mathrm{PAH}} / L_{\mathrm{TIR}}=0.1$ for both $\mathrm{H}$ II and AGN galaxies. At the highest relative PAH luminosities, systems dominated by star formation predominate (at $L_{\mathrm{PAH}} / L_{\mathrm{TIR}}>0.12$, only 3 out of 14 systems host AGNs), despite the fact that the spectral apertures include both nuclear and inner disk emission. This may suggest a natural limit on the absolute PAH strength in sources with weak AGNs, due to either partial destruction of the grains or the contribution of other continuum sources to the total infrared luminosity.

Figure 11 demonstrates the large scatter in the ratio $L(\mathrm{PAH}) /$ $\nu L_{\nu}(24 \mu \mathrm{m})$ for the four strongest PAH features as a function of the local far-infrared color temperature $\nu L_{\nu}(24 \mu \mathrm{m}) /$ $\nu L_{\nu}(70 \mu \mathrm{m})$. The full range is typically over a factor of 10 for each band and may in fact be a lower limit on the true scatter in the global emission of galaxies, for which diffuse scattered light in the outer regions can account for a substantial fraction of the $24 \mu \mathrm{m}$ luminosity (Calzetti et al. 2005). For all bands, there is a definite negative trend of $L(\mathrm{PAH}) / \nu L_{\nu}(24 \mu \mathrm{m})$, which suggests that a mix of both small stochastically heated and thermally emitting grains contributes to the $24 \mu \mathrm{m}$ continuum, perhaps due to varying contributions from PDR and $\mathrm{H}$ II regions (e.g., Laurent et al. 2000).

TABLE 6

PAH Feature Luminosity Estimates: Full Decomposition versus Spline Ratios

\begin{tabular}{|c|c|}
\hline Feature & $L($ decomp $) / L($ spline $)$ \\
\hline ......................... & $1.70 \pm 0.42$ \\
\hline 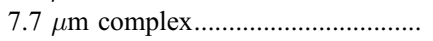 & $3.53 \pm 0.62$ \\
\hline $8.6 \mu \mathrm{m}$ & $3.31 \pm 0.64$ \\
\hline $11.3 \mu \mathrm{m}$ complex & $1.86 \pm 0.15$ \\
\hline
\end{tabular}




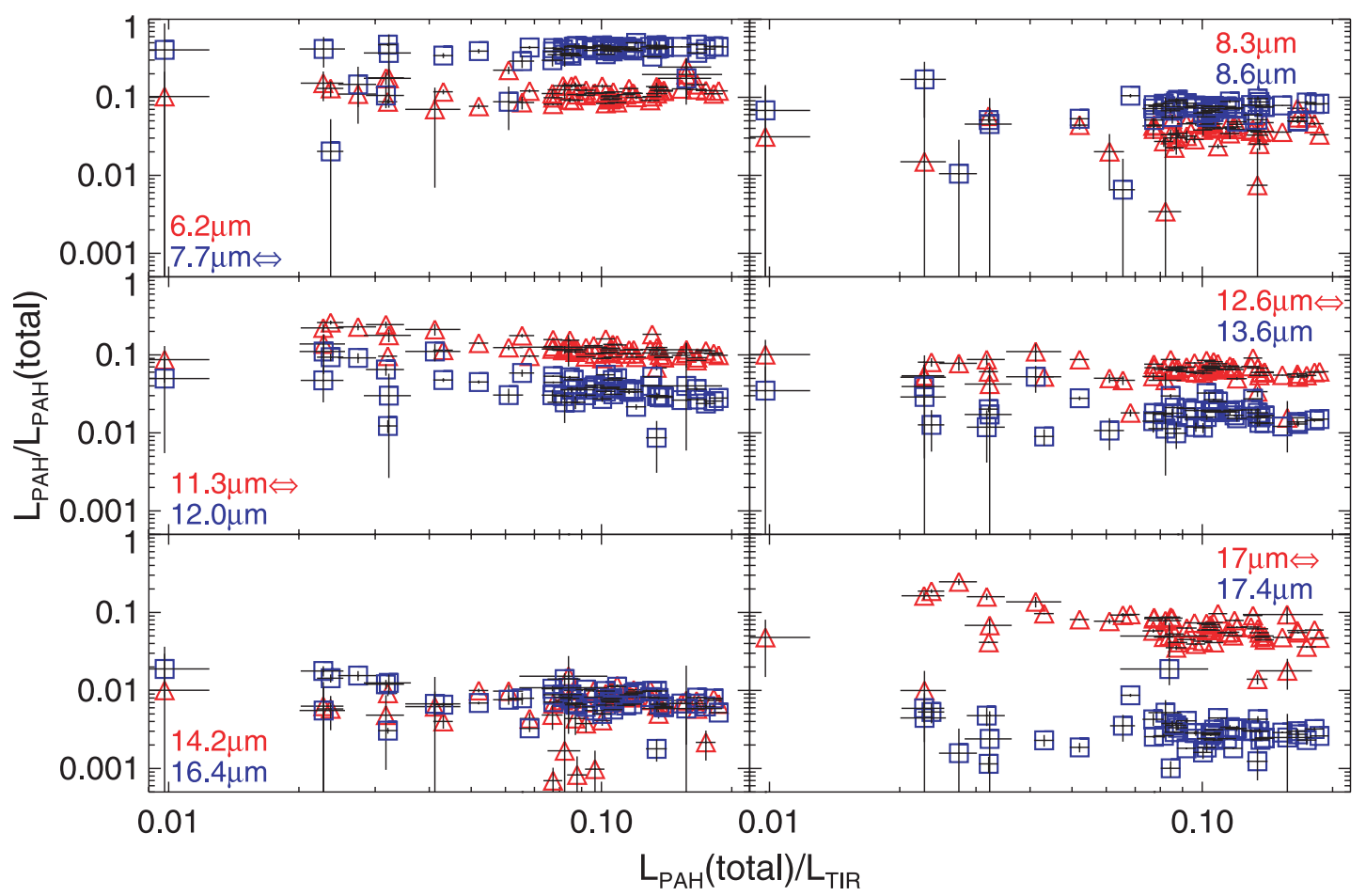

FIG. 9.-Fractional PAH luminosity relative to the total PAH power for the main features identified in the spectra, against the integrated PAH contribution to the total infrared luminosity from 3 to $1100 \mu \mathrm{m}$, in the same region. Blended complexes are marked by a double arrow in the legend; see Table 3 .

\subsection{The PAH Contribution to IRAC $8 \mu \mathrm{m}$ and MIPS $24 \mu \mathrm{m}$}

With the full spectral decomposition of continuum and dust features, we can examine the fractional contribution of PAH emission and stellar or thermal dust continuum to the broad Spitzer bandpasses. We consider IRAC $8 \mu \mathrm{m}$ at $z=0$ and MIPS $24 \mu \mathrm{m}$ at $z=2$. These broad bands are commonly used as surrogates for PAH strength in nearby and distant galaxy surveys (e.g., Hogg et al. 2005; Papovich et al. 2006; Reddy et al. 2006). Since IRAC $8 \mu \mathrm{m}$ at low redshift and MIPS $24 \mu \mathrm{m}$ at $z \sim 2$ encompass the 7.7 and $8.6 \mu \mathrm{m}$ PAH bands, typically the strongest (although see $\S 5.6$ ), a common assumption is that broadband photometry at these rest wavelengths serves as a direct proxy for PAH emission, total infrared or bolometric luminosity, and, potentially, star formation rate. For low-redshift sources, pre-

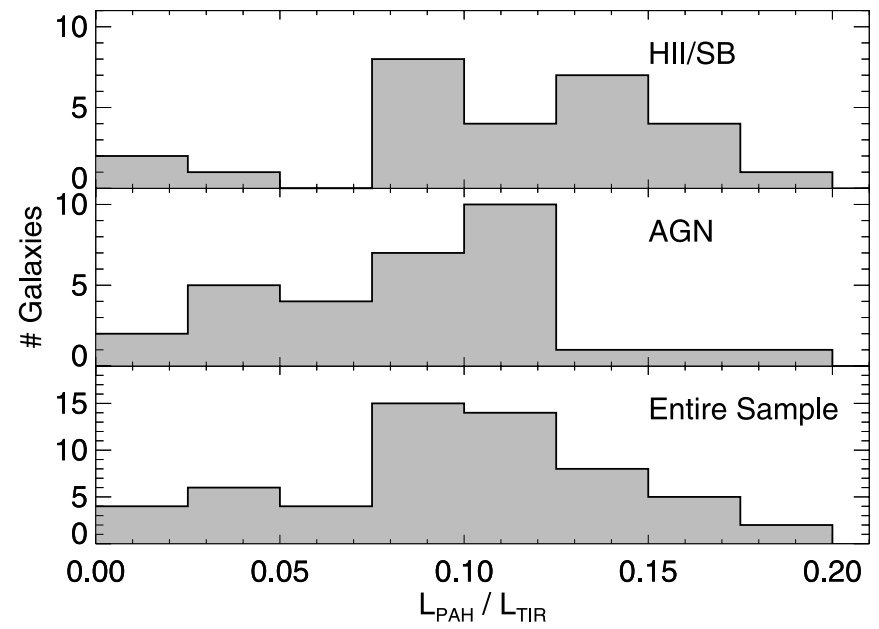

FIG. 10.-Distribution of the integrated PAH luminosity relative to the total infrared, $L_{\mathrm{PAH}} / L_{\mathrm{TIR}}$, for the entire sample, and individually for galaxies with AGN and $\mathrm{H}$ II/starburst classifications. scriptions exist for removing the stellar contribution using the 3-5 $\mu \mathrm{m}$ channels of IRAC (e.g., Helou et al. 2004). The relatively isolated $24 \mu \mathrm{m}$ band does not typically permit such starlight removal (although the IRS peak-up blue channel at $16 \mu \mathrm{m}$ could in principle serve this purpose).

Figure 12 highlights the large fractional contribution of PAH feature emission to the uncorrected IRAC $8 \mu \mathrm{m}$ band at $z=0$ and MIPS $24 \mu \mathrm{m}$ band at $z=2$. The median contribution for both is $\sim 0.7$, with a small number of sample members (sources dominated by stellar continuum) showing very little PAH contribution. For over $75 \%$ of the sample, PAH emission contributes more than one-half of the broadband $8 \mu \mathrm{m}$ power. If the stellar continuum is perfectly removed, the distribution shifts only slightly to higher fractional PAH contribution.

The typical source in deep MIPS $24 \mu \mathrm{m}$ surveys has total infrared luminosity $L_{\mathrm{TIR}} \gtrsim 10^{11} L_{\odot}$ (Papovich et al. 2006; Yan

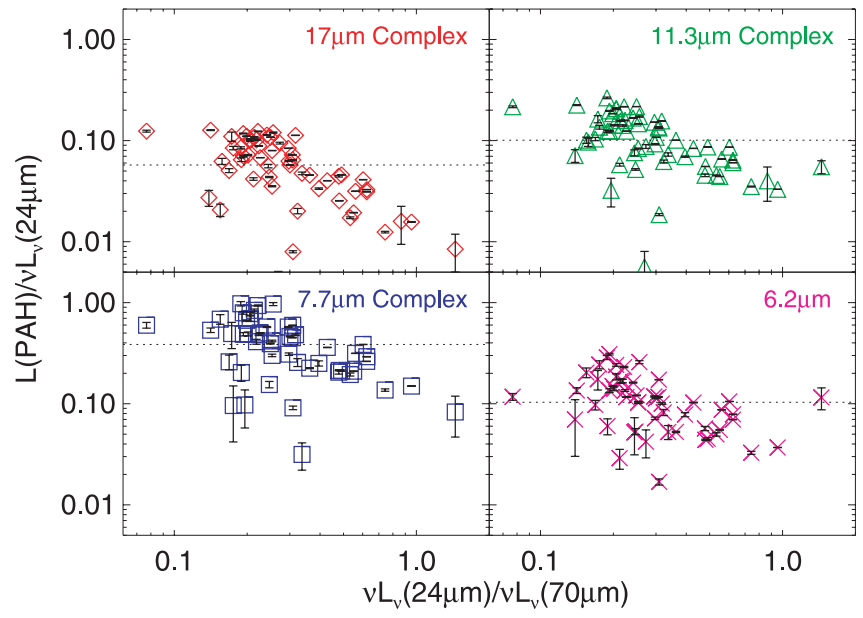

FIG. 11.-PAH-to-MIPS $24 \mu \mathrm{m}$ luminosity ratio vs. far-infrared color for the four strongest PAH bands, in matched apertures. 


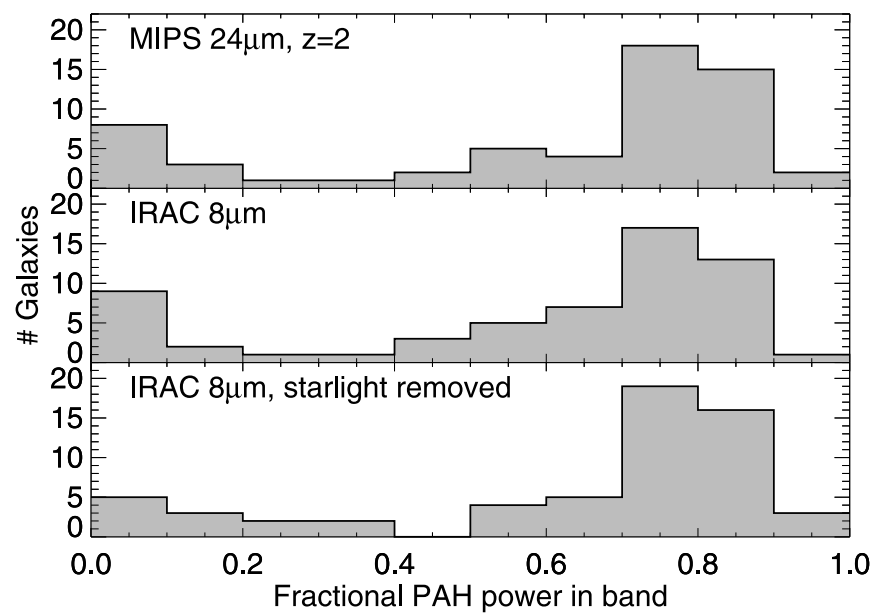

FIG. 12.-Distribution among the sample of the fractional contribution of dust features to the Spitzer broad bands, for IRAC $8 \mu \mathrm{m}$ at redshift $z=0$, with and without stellar continuum removed from the band, as well as MIPS $24 \mu \mathrm{m}$ at $z=2$.

et al. 2004) and may therefore exhibit a much different contribution of PAH emission in the $8 \mu \mathrm{m}$ rest frame compared to starforming galaxies of lower luminosity. The sample explored here has median global $L_{\mathrm{TIR}}$ of $0.8 \times 10^{10} L_{\odot}$. It should also be noted that the dominance of PAH emission in the band, e.g., MIPS $24 \mu \mathrm{m}$ at $z \sim 2$, does not imply anything about the efficacy of using this single photometric point to derive the total infrared luminosity or star formation rate (see Dale et al. 2005).

\subsection{PAH Strength Variations}

Figure 13 demonstrates the variation in interband strength ratios over the full sample for the four strongest $\mathrm{PAH}$ bands at $6.2,7.7,11.3$, and $17 \mu \mathrm{m}$. Both the $6.2 \mu \mathrm{m} / 7.7 \mu \mathrm{m}$ and $17 \mu \mathrm{m} /$ $11.3 \mu \mathrm{m}$ ratios vary by more than a factor of 2 . Sources with weak AGNs are almost entirely offset from sources with $\mathrm{H}$ II-like nuclear optical spectra, an effect that is explored in the next section. Sources with nonnegligible mixed silicate absorption optical depths (circled) are offset slightly from the main locus of points for their type ( $\mathrm{H}$ II or AGN), to lower values of $L(6.2 \mu \mathrm{m}) / L(7.7 \mu \mathrm{m})$ and $L(11.3 \mu \mathrm{m}) / L(17 \mu \mathrm{m})$. This is in the direction of increased reddening, despite the implicit dereddening performed by the spectral decomposition method, and could be evidence of different absolute extinctions for the underlying continuum and the PAH-emitting regions, or of limitations in the adopted extinction curve.

The medians and 10\%-90\% range of variation for luminosity ratios among the most important PAH bands, the integrated PAH luminosity, and the total infrared luminosity are given in Table 7. Typical variations in the interband luminosities are a factor of $2-5$, with $1 \sigma$ variations in these ratios of $\pm 30 \%$.

\subsubsection{PAHs in AGNS}

Nuclear black holes are now recognized as a fundamental property of many local elliptical and spiral galaxies: $\gtrsim \frac{1}{3}$ have optical nuclear spectra typical of low-luminosity AGNs (Ho et al. 1995, 1997). The SINGS sample was not defined to include powerful AGN sources, yet roughly half of the galaxies considered here show some form of AGN activity in their nuclear

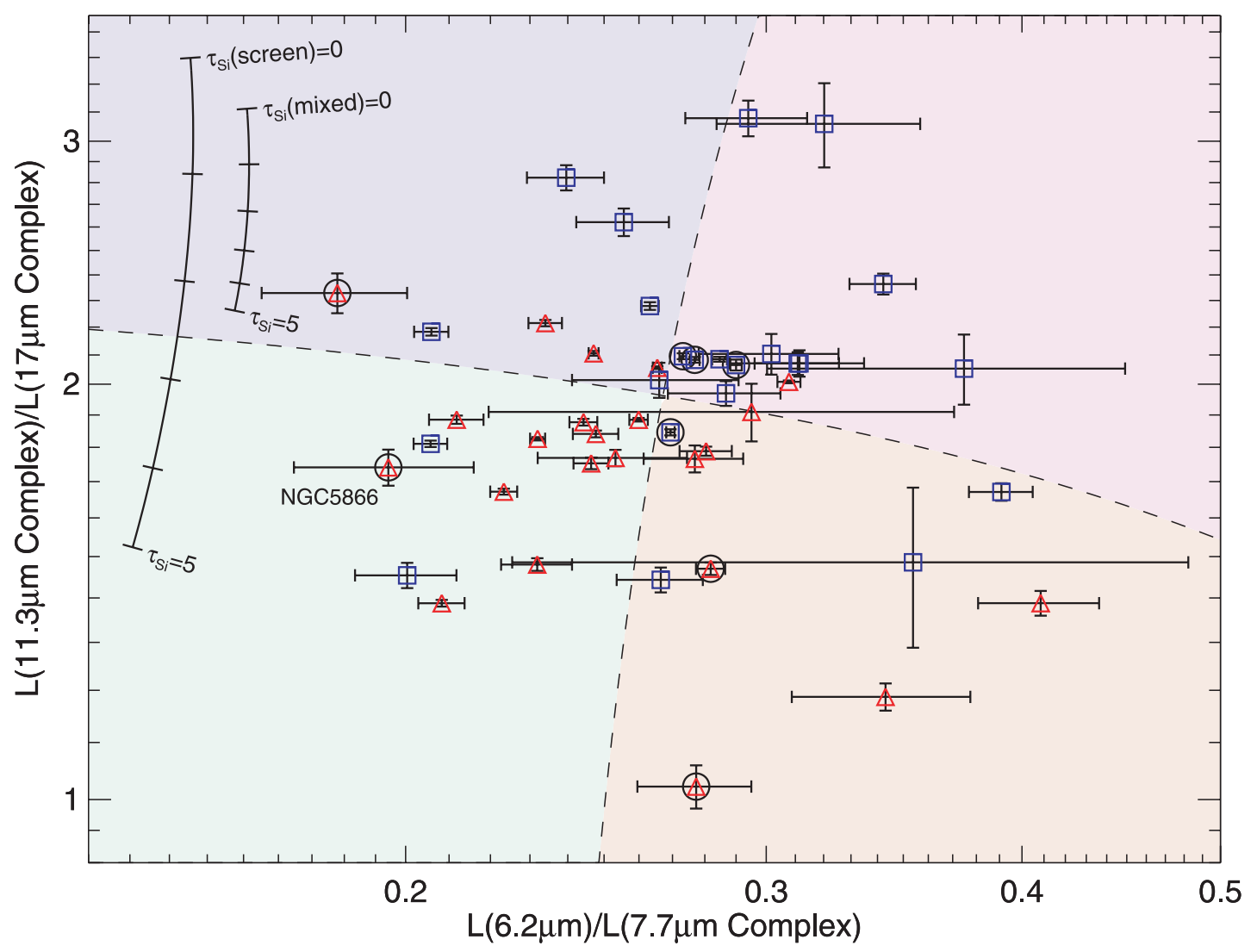

FIG. 13.--Interband strength ratios of the four main PAH bands at 6.2, 7.7, 11.3, and $17 \mu$ m. Red triangles indicate galaxies with AGN (Seyfert or LINER) types,

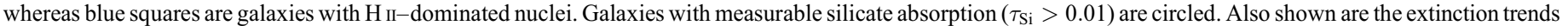

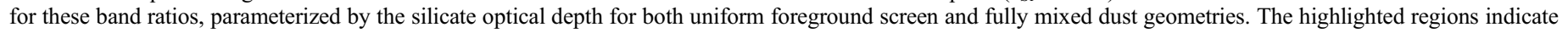
zones used for the construction of template spectra (see $\S 6$ ). 
TABLE 7

PAH Band Luminosity Ratios $L\left(\lambda_{1}\right) / L\left(\lambda_{2}\right)$

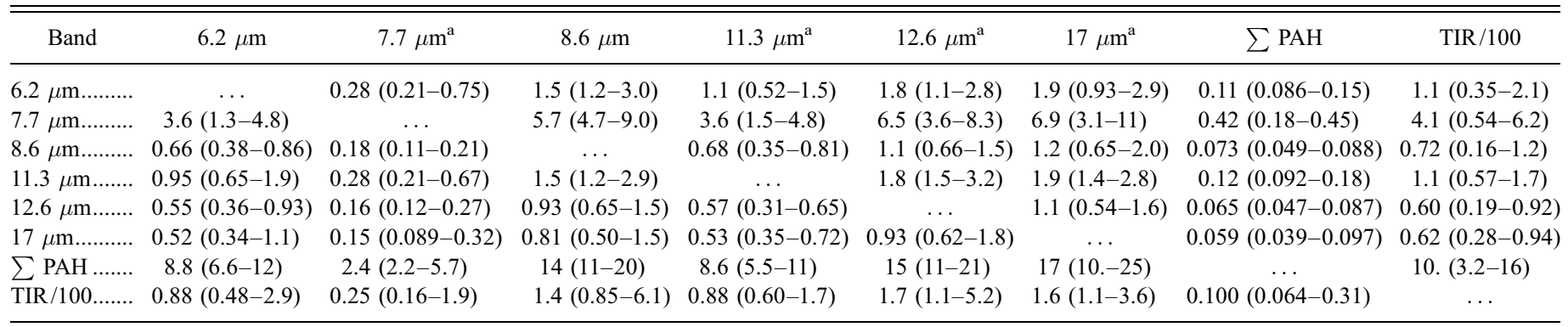

NoтE.-Each entry is encoded as med (low-high), where med is the median of the ratio, and low-high is the $10 \%-90 \%$ range of variation.

a Indicates blended PAH complexes; see Table 3 for components.

optical spectroscopy. The SINGS AGN sources are in the lowluminosity regime, with $L\left([\mathrm{O}\right.$ III $]$ 25007) $<10^{6.5} L_{\odot}$ [compared to $L([\mathrm{O} \mathrm{III}]) \sim 10^{7}-10^{9} L_{\odot}$ for traditional AGNs; Kewley et al. 2006]. This sample makes it possible to test the behavior of PAH emission in the presence of weak AGNs.

In Figure 14 the ratio of two strong PAH bands is compared to the hardness indicator [ $\mathrm{Ne} \mathrm{III}] /[\mathrm{Ne} \mathrm{II}]$, with an ionization potential ratio of $41 \mathrm{eV} / 21.6 \mathrm{eV}$. Galaxies with $\mathrm{H}$ in region or starburstlike optical spectra exhibit a nearly constant $L(7.7 \mu \mathrm{m}) / L(11.3 \mu \mathrm{m})$ ratio across the full range of radiation hardness, including those low-metallicity systems (when PAHs are detected) with [Ne III]/ $[\mathrm{Ne}$ II $] \gtrsim 2$. In striking contrast, the AGNs as a group are offset below the locus of $\mathrm{H}$ II-type galaxies, even at moderate hardness ratios, and at $[\mathrm{Ne} \mathrm{III}] /[\mathrm{Ne}$ II $] \sim 1$ fall away rapidly. Among the 10 galaxies in the sample with the lowest limiting band ratios
$L(7.7 \mu \mathrm{m}) / L(11.3 \mu \mathrm{m})$, all show evidence of low-luminosity LINER or Seyfert activity. In these systems, the relative strength of the short-wavelength PAH emission bands is suppressed by up to a factor of 10, compared to star formation-dominated systems. An example of such a spectrum is shown in Figure 15, for the LINER galaxy NGC 1316, with the predicted spectrum for the sample's median band ratios overlayed.

One effect that could lead to the observed behavior is ionization state. Since the $11.3 \mu \mathrm{m}$ feature is thought to be produced by neutral PAHs, whereas the $7.7 \mu \mathrm{m}$ feature arises primarily from PAH cations (e.g., Allamandola et al. 1999; Li \& Draine 2001), a change in the neutral fraction could impact their relative strengths. Harder radiation fields are also more efficient at destroying PAH grains, an effect that may dominate the observed trend, if the grain or molecular carriers of the 7.7 and $11.3 \mu \mathrm{m}$

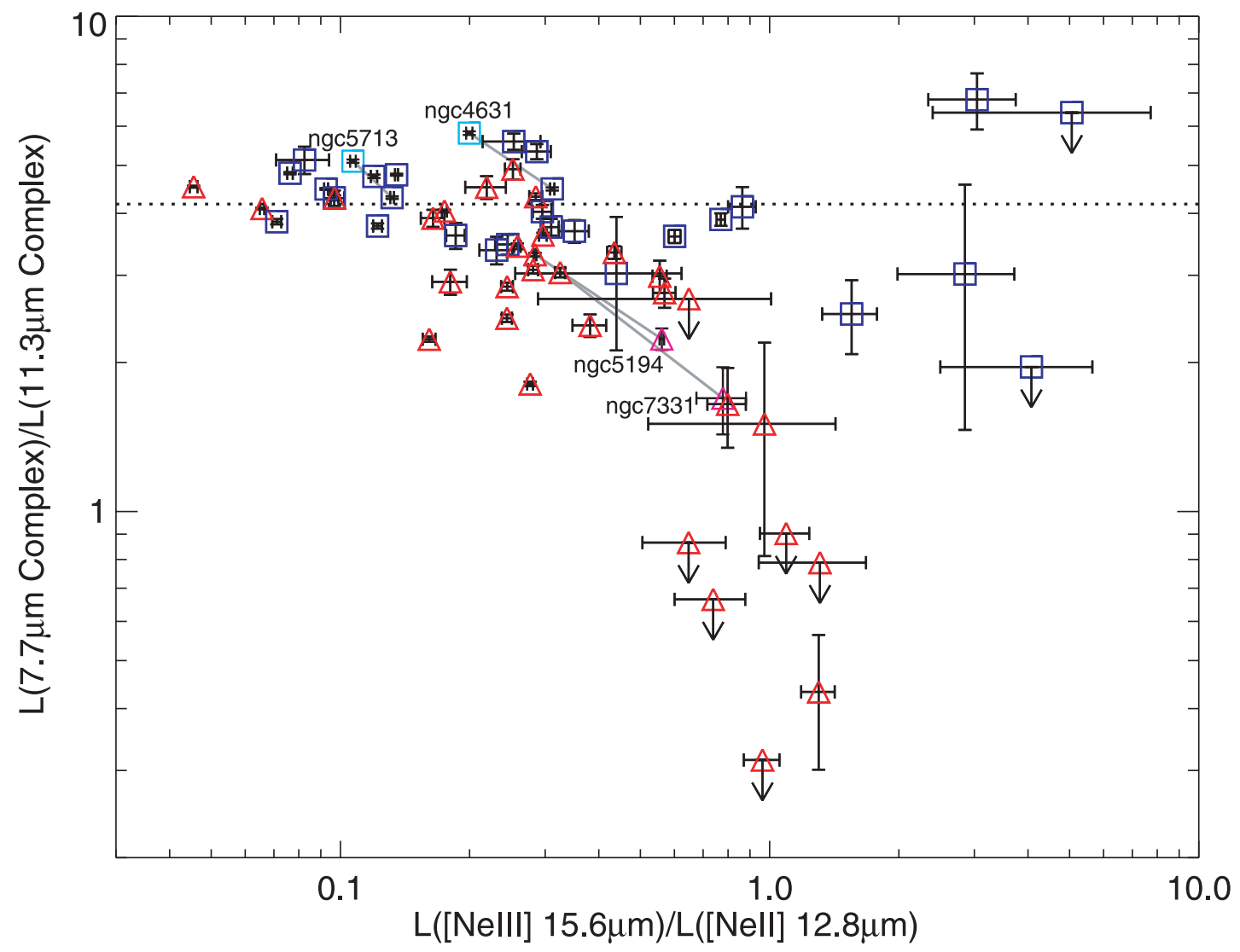

FIG. 14. - Variation of interband strength ratios of the PAH features at $7.7 \mu \mathrm{m}$ relative to the $11.3 \mu \mathrm{m}$ band with the line ratio [Ne III]/[Ne II], an indicator of the hardness of the radiation field. Red triangles indicate galaxies with AGN (Seyfert or LINER) types, whereas blue squares are galaxies with $\mathrm{H}$ II-dominated nuclei. Upper limits are $2 \sigma$. The dotted line shows the median of the $\mathrm{H}$ II-dominated sources. Four sources (two AGNs, magenta triangles; two $\mathrm{H}$ II nuclei, cyan squares) are plotted with additional values obtained from spectra extracted over small $9 \operatorname{arcsec}^{2}$ apertures centered on their nuclei and connected by solid gray lines to their larger aperture values. 


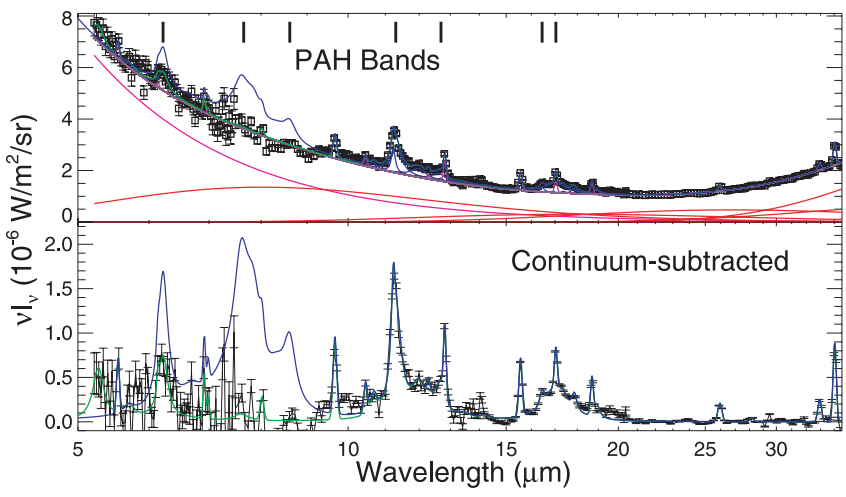

FIG. 15.-Example of the peculiar low-luminosity AGN PAH emission spectrum in barred LINER galaxy NGC 1316, with the full decomposition shown as the green solid line. The PAH spectrum predicted from the strength of the $11.3 \mu \mathrm{m}$ features and adapted to the SINGS median band ratios is shown in blue, with a continuum-subtracted version below.

bands are different, and undergo photodestruction via ionization or dissociation at substantially differing rates. The $11.3 \mu \mathrm{m}$ feature can be produced by single-photon heating of PAHs that are larger than those that are effective at producing $7.7 \mu \mathrm{m}$ emission (e.g., Schutte et al. 1993; Draine \& Li 2006). The observed decrease in $L(7.7 \mu \mathrm{m}) / L(11.3 \mu \mathrm{m})$ may then be the result of selective destruction of those PAHs small enough to emit at $7.7 \mu \mathrm{m}$. Low-metallicity dwarfs with the highest [ $\mathrm{Ne}$ III]/[ $\mathrm{Ne}$ II $]$ ratios appear to have roughly normal band ratios (when PAHs are detected), suggesting that AGNs must play a role.

Further evidence comes from a related trend in spatially resolved spectral maps of large SINGS spiral galaxies with AGN nuclei. The $7.7 \mu \mathrm{m} / 11.3 \mu \mathrm{m}$ band ratio in many cases decreases noticeably in the nucleus, compared to larger apertures that sample star-forming regions in the inner disk. An example of this effect for four galaxies chosen at $d \lesssim 8 \mathrm{Mpc}$ with both bright inner disk and nuclear emission is illustrated in Figure 14. Ratios found in the default apertures are connected by solid lines to those recovered in small $9.25^{\prime \prime} \times 9.25^{\prime \prime}$ (roughly $300 \mathrm{pc}$ ) apertures centered on the nucleus. For the two systems with AGNs (NGC 5194 and NGC 7331), the short-wavelength PAH features show further suppression in their nuclear spectra, whereas the $\mathrm{H}$ in galaxies (NGC 4631 and NGC 5713) move to slightly higher values of $L(7.7 \mu \mathrm{m}) /$ $L(11.3 \mu \mathrm{m})$. Such spatially resolved variations will be considered in more detail in a subsequent paper but are consistent with the interpretation that the hard radiation fields near AGNs have a strong impact on the strength of the short-wavelength PAH bands.

Kaneda et al. (2005) note an unusually weak $7.7 \mu \mathrm{m}$ feature in IRS spectra of three X-ray-bright elliptical galaxies, as well as SINGS elliptical NGC 4125 (which has the fourth lowest PAH band ratio in Fig. 14). They suggest that unusual ISM properties in evolved elliptical galaxies could be responsible. However, two of these three elliptical galaxies have established optical AGN activity (NGC 2974, a Seyfert 2, and NGC 4589, a LINER), and our own recently obtained nuclear optical spectra of the third, NGC 3962, indicates that it also hosts an unambiguous AGN. This suggests that the AGN environment, rather than the global morphological type, leads to the unusual emission characteristics. Elliptical galaxies, with little contamination from the PAH emission associated with ongoing star formation, may simply offer the most favorable systems for detecting this unusual spectrum. Consistent with this, Brandl et al. (2006) found a large scatter but no significant variation of $L(7.7 \mu \mathrm{m}) / L(11.3 \mu \mathrm{m})$ over a wide range of excitation in starburst nuclei.
An alternative explanation is that the AGN is unrelated to or only partially responsible for the change in the PAH spectrum. This would be the case if AGN classification acts merely as a surrogate of low star formation intensity, since strong nuclear star formation can overwhelm the AGN diagnostic lines. Cesarsky et al. (1998) found a depressed $7 \mu \mathrm{m}$ band in differential ISOCAM spectra within M31, suggesting that this could be due to the weak UV field. In Spitzer samples of 50 passively evolving earlytype galaxies (Bregman et al. 2006; Bressan et al. 2006), only two quiescent sources show PAH emission, one of which, NGC 4550, hosts a bright LINER nucleus and exhibits the same strong $11.3 \mu \mathrm{m}$ feature we report. The other, NGC 4697, is without an apparent AGN yet also shows a moderately depressed $7 \mu \mathrm{m}$ PAH, although Bregman et al. (2006) conclude that after removing silicate emission, a spectrum similar to the diffuse ISM of the Galaxy is recovered. Future studies will better constrain the role of AGNs and weak star formation intensity in the modification of the PAH emission spectrum.

If AGNs dominate the effect, the absence of PAH emission in nearly all quiescent galaxies suggests that, especially in systems with little ongoing star formation, the AGN itself could excite PAH emission, supplying UV radiation dominated by Ly $\alpha$ and $\mathrm{H}_{2}$ Lyman-Werner bands in the extended X-ray-dominated regions (Meijerink \& Spaans 2005), with a hardness spectrum quite unlike that typically found in environments harboring young stellar populations. In addition, an AGN may emit X-rays sufficient to destroy small PAHs directly even at distances of kiloparsecs (Voit 1992). Because larger PAHs are expected to be less susceptible to destruction by X-rays, the presence of an AGN can alter the PAH emission spectrum, even if the PAH excitation itself is dominated by starlight from an older stellar population. Clearly, the absolute luminosity density of the AGN source is also an important factor regulating the PAH spectrum, since more luminous AGNs either destroy all PAH grains (e.g., Voit 1992; Lutz et al. 1998) or produce MIR continuum photons in such copious quantities that the PAH bands are diluted away (Siebenmorgen et al. 2005; Hao et al. 2005; Sturm et al. 2005). This effect was exploited by Genzel et al. (1998) to differentiate between AGNs and starbursts at high luminosity. Although grain size modification may operate in many contexts, the unusual band ratios observed in low-luminosity AGN nuclei $[L(7.7 \mu \mathrm{m}) / L(11.3 \mu \mathrm{m}) \lesssim 2]$ could offer an efficient way to detect such weak active nuclei, when the UV field is not dominated by star formation and dust absorption hinders optical classification. Although it is not yet known over what regimes this effect operates, the possibility that AGN sources could directly excite PAH emission suggests considerable caution when using the PAH bands as direct indicators of star formation in systems known to harbor active nuclei.

Care must be taken to quantify the band ratios that constitute an unusual PAH spectrum of this type, since the $11.3 \mu \mathrm{m}$ feature, while not the most luminous, has the highest specific luminosity per wavelength interval and does not typically suffer continuum dilution from starlight as the shorter wavelength PAH features can. At low signal and/or with strong stellar continuum, the $11.3 \mu \mathrm{m}$ feature can remain the most easily detectable PAH band and appear to dominate despite normal band ratios. An example of this phenomenon is found in the SINGS dwarf galaxy NGC 1705 (Cannon et al. 2006).

\subsubsection{PAHs and Metallicity}

That the absolute strength of PAH emission can depend on metallicity has recently been well established (Engelbracht et al. 


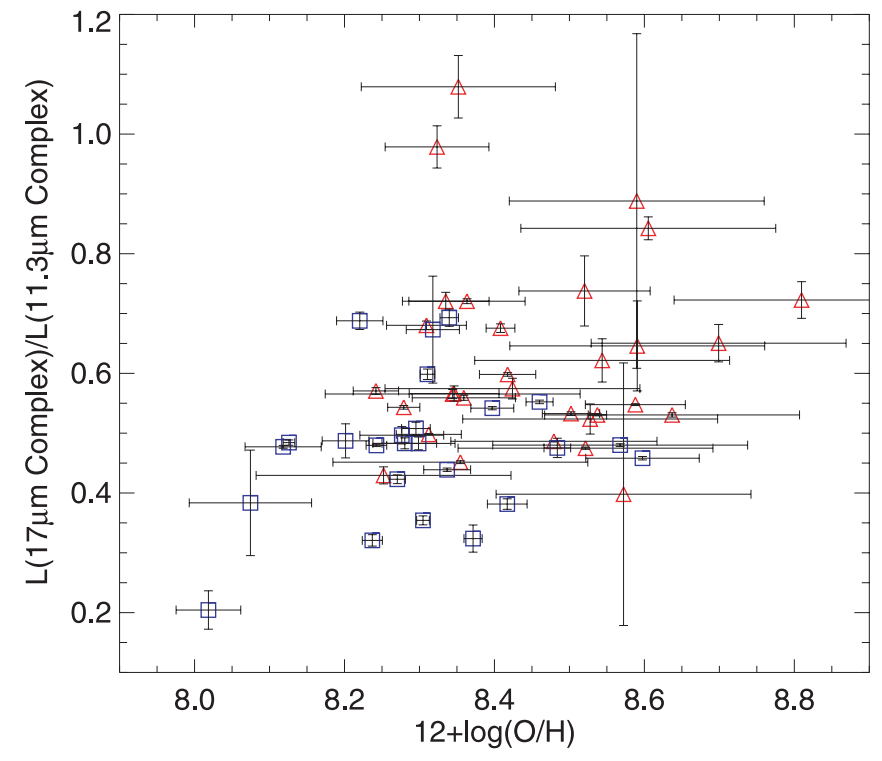

FIG. 16.-Variation of the $17 \mu \mathrm{m} / 11.3 \mu \mathrm{m}$ PAH luminosity ratio with oxygen abundance. Red triangles are sources with LINER/Seyfert classifications, while blue squares have $\mathrm{H}$ II classifications.

2005; Madden et al. 2006; Wu et al. 2006; O'Halloran et al. 2006; Rosenberg et al. 2006), with indications of a threshold $12+\log (\mathrm{O} / \mathrm{H}) \sim 8.1$, or approximately $Z_{\odot} / 4$, below which PAH emission is suppressed. From detailed SED fitting of the SINGS sample, B. T. Draine et al. (2007, in preparation) find that the fraction of the dust mass contributed by PAHs is suppressed below a similar threshold. Most studies rely on Spitzer broadband photometry (e.g., $8 \mu \mathrm{m} / 24 \mu \mathrm{m}$ ) to assess absolute PAH strengths. Although broadband $8 \mu \mathrm{m}$ flux does correlate well with PAH strength (see $\S 5.5$ ), the range of MIR continuum properties can overwhelm even significant changes in the relative PAH luminosity; see, e.g., the large variation in $7.7 \mu \mathrm{m} \mathrm{PAH} \mathrm{to}$ $24 \mu \mathrm{m}$ luminosity in Figure 11. Broadband methods are also not sensitive to changes in the form of the PAH emission spectrum.

Figures 16 and 17 illustrate two examples of the behavior of PAH emission with changes in nuclear oxygen abundance: the relative strengths of the 17 and $11.3 \mu \mathrm{m}$ PAH bands, and the total integrated PAH luminosity relative to the total infrared. Oxygen abundances for the SINGS galaxies have been determined by J. Moustakas et al. (2007, in preparation), based on a combination of nuclear and circumnuclear optical spectroscopy and observations of individual $\mathrm{H}$ II regions culled from the literature and placed on the common $12+\log (\mathrm{O} / \mathrm{H})$ strong-line calibration scale of Pilyugin \& Thuan (2005).

The central spectra of SINGS galaxies probe much higher oxygen abundance than the low- $Z$ sample of Engelbracht et al. (2005), yet, even at these relatively high metallicities, up to $\sim 1.5 Z_{\odot}$, there is a definite trend in the interband strength ratios, with higher metallicity leading to increased $L(17 \mu \mathrm{m}) /$ $L(11 \mu \mathrm{m})$. Although low-metallicity systems do not tend to harbor AGNs (Kauffmann et al. 2003), the presence of a weak AGN in the nucleus clearly has a strong impact on the behavior of $L(17 \mu \mathrm{m}) / L(11 \mu \mathrm{m})$, with the highest ratios observed only in systems with active nuclei.

Since the 15-20 $\mu \mathrm{m}$ PAH bands are hosted by larger grains than the shorter wavelength features, the trend with metallicity is suggestive of a scenario in which formation of the large grains that contribute the $17 \mu \mathrm{m}$ band is enhanced in the presence of higher metal abundance. This effect could act in combination

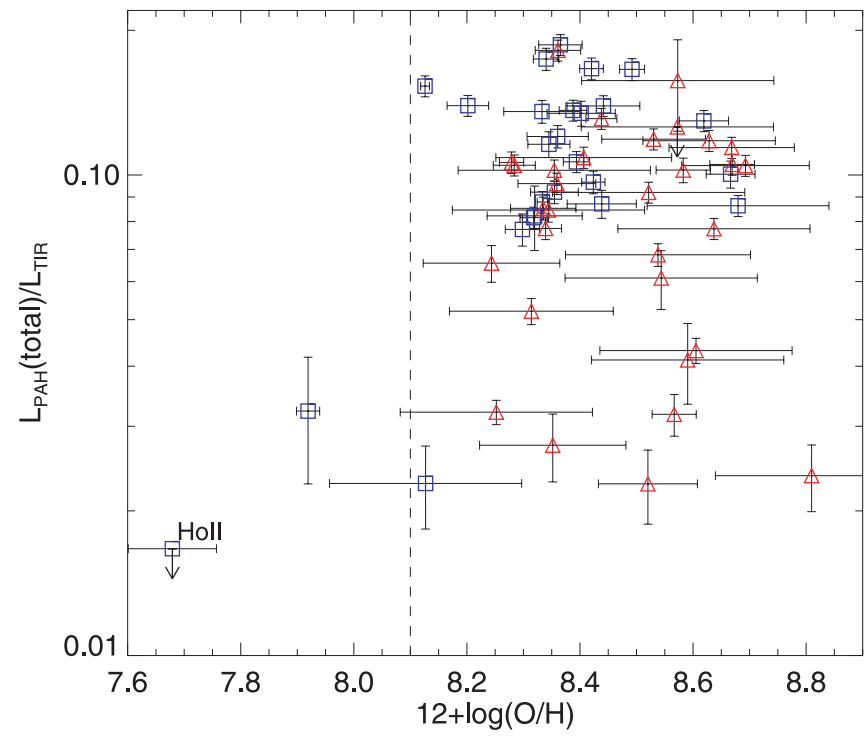

FIG. 17.- Variation of the total PAH luminosity relative to the total infrared $(3-1100 \mu \mathrm{m})$ luminosity. The threshold abundance $12+\log (\mathrm{O} / \mathrm{H})=8.1$ is indicated. Symbols are as in Fig. 16.

with the destruction of smaller PAH grains in AGN environments, since it is likely that the mechanism that suppresses the shortest wavelength PAH features in AGNs has a similar, if smaller, effect on the $11.3 \mu \mathrm{m}$ feature.

Low-metallicity systems also have harder radiation fields due to a combination of two effects: (1) hotter massive stars form in low-metallicity environments (an effect dominated by the metallicity sensitivity of line-driven stellar winds; MartínHernández et al. 2002), and (2) dust production is inhibited, leading to reduced shielding by dust grains. This could also impact the PAH band ratios of Figure 16. However, since both the 11.3 and $17 \mu \mathrm{m}$ bands are contributed by neutral PAHs, the ionization state cannot drive the trend in their ratios. Likewise, selective destruction of the smaller grains in the harder fields would result in the opposite trend: higher $L(17 \mu \mathrm{m}) / L(11.3 \mu \mathrm{m})$ at lower metallicity. This leaves the inhibited formation of the largest grains at low metallicity as the most likely dominant effect. Clearly, however, metallicity variation alone cannot explain the full range of $L(17 \mu \mathrm{m}) / L(11.3 \mu \mathrm{m})$ in Figure 16, since at $Z \gtrsim Z_{\odot} / 2$ AGNs consistently have the largest ratio.

The behavior of the total PAH luminosity, integrated over all bands from 5 to $19 \mu \mathrm{m}$, relative to the total infrared luminosity in Figure 17 shows a similar trend, with high fractional PAH contribution to the total infrared ( $\gtrsim 10 \%)$ occurring only at metallicities above $12+\log (\mathrm{O} / \mathrm{H}) \sim 8.1$. There is also a marked separation between galaxies with weak AGNs and H II classifications. At a given metallicity (even supersolar), AGN sources emit a much smaller fraction of their central infrared luminosity as PAH emission. Coupled with the anomalous PAH band ratios seen in the most extreme such sources (Fig. 14), a picture of weak AGNs profoundly influencing the absolute strength and shape of the PAH emission spectrum emerges.

\section{SED MODELS IN THE HIGH-REDSHIFT CONTEXT}

Rather than directly modeling the physical processes and resulting MIR emission properties of star-forming galaxies, most broadband infrared studies of moderate- to high-redshift galaxies have utilized infrared templates, constructed from small samples of local galaxy spectra. Since the PAH bands are so important energetically and impart the 3-19 $\mu \mathrm{m}$ spectrum of 


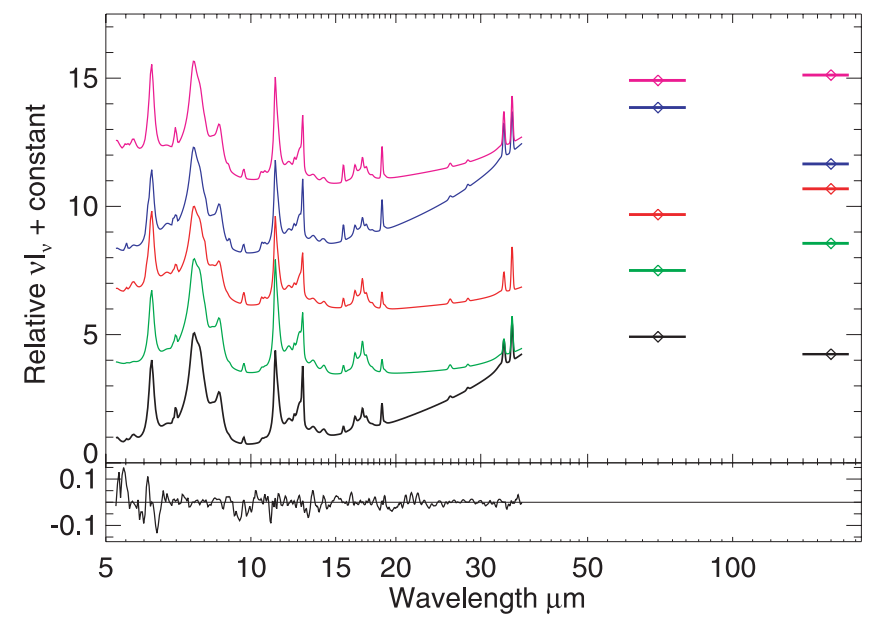

FIG. 18.-Noise-free MIR templates, produced by a TIR luminosity-weighted average of all spectra (black) and from subsample means for each region in Fig. 13 (color). The units are arbitrary $\nu I_{\nu}$, with a constant offset of 2.5 units between spectra. Mean matched 70 and $160 \mu \mathrm{m}$ intensities are also shown. The bottom panel contains the normalized residuals (model-spectrum)/model, averaged over the sample, with $20 \%$ trimmed from the extremes of the distribution.

star-forming galaxies with features that contrast strongly with each other and with the underlying dust and stellar continuum, they are a vital component of any infrared SED template. A small number of bright, very well studied systems with high-quality ISO spectra (e.g., Arp 220, M82, NGC 253, Mrk 231; Rigopoulou et al. 1999; Sturm et al. 1996, 2000) have been used extensively (e.g., Elbaz et al. 2002; Webb et al. 2006; Reddy et al. 2006). The average ISOPHOT spectrum of Lu et al. (2003) has also seen wide use, having been integrated into the SED library of Dale et al. (2001) and others. Even physically realistic SED models (e.g., Silva et al. 1998) are often used with the MIR substituted by scaled template spectra.

The importance of quantifying not just the average shape of the MIR spectrum of star-forming galaxies but the range of variation in that shape is well illustrated by the source count models of Lagache et al. (2004). To model deep MIPS $24 \mu \mathrm{m}$ source counts (Papovich et al. 2004), Lagache et al. (2004) apply a modest tilt to the ISO PAH template spectrum to enhance the power at $15 \mu \mathrm{m}$. With this modified SED, the source counts, which could not be reproduced by preexisting models, came into very good agreement. This perturbation played a large role in the interpretation of the redshift distribution and star formation history implied by the broadband survey sample. Such sensitivity to the detailed form and shape of the MIR emission spectrum underlines the urgent need to quantify variations in this spectrum and establish the role of such natural variations in the interpretation of resolved and unresolved survey results.

In Figure 18, five noiseless model templates from the spectral decomposition technique of $\S 4$ are shown: an average spectrum weighted by the global total infrared luminosity, and four subsample averages drawn from the empirically defined regions in Figure 13, formed by lines in the $L(6.2 \mu \mathrm{m}) / L(7.7 \mu \mathrm{m})$ versus $L(11.3 \mu \mathrm{m}) / L(17 \mu \mathrm{m})$ space. These boundaries were chosen to ensure adequate sampling in each region and to bracket a modest range of variation within the sample. Only galaxies with reliable detection in all four main PAH bands are included (eliminating all sources dominated by stellar continuum). Emission lines are not removed. The region-averaged spectra corresponding to the lower two quadrants in Figure 13 have the largest contribution from weak AGN sources. The normalized residuals are also shown
TABLE 8

NoISE-Free MIR Template Spectra

\begin{tabular}{cccccc}
\hline \hline $\begin{array}{c}\text { Wavelength } \\
(\mu \mathrm{m})\end{array}$ & $\begin{array}{c}\text { Temp1 } \\
\left(f_{\nu}\right)\end{array}$ & $\begin{array}{c}\text { Temp2 } \\
\left(f_{\nu}\right)\end{array}$ & $\begin{array}{c}\text { Temp3 } \\
\left(f_{\nu}\right)\end{array}$ & $\begin{array}{c}\text { Temp4 } \\
\left(f_{\nu}\right)\end{array}$ & $\begin{array}{c}\text { Luminosity Weighted } \\
\left(f_{\nu}\right)\end{array}$ \\
\hline $5.2469 \ldots \ldots \ldots \ldots .$. & 0.0313 & 0.0249 & 0.0447 & 0.0150 & 0.0170 \\
$5.2861 \ldots \ldots \ldots \ldots .$. & 0.0316 & 0.0250 & 0.0449 & 0.0152 & 0.0173 \\
$5.3253 \ldots \ldots \ldots \ldots .$. & 0.0311 & 0.0248 & 0.0433 & 0.0148 & 0.0164 \\
$5.3644 \ldots \ldots \ldots \ldots .$. & 0.0306 & 0.0247 & 0.0417 & 0.0143 & 0.0154 \\
$5.4036 \ldots \ldots \ldots \ldots \ldots$ & 0.0304 & 0.0247 & 0.0408 & 0.0141 & 0.0149
\end{tabular}

Notes.-Table 8 is published in its entirety in the electronic edition of the Astrophysical Journal. A portion is shown here for guidance regarding its form and content.

and are typically only a few percent, with some structure near known artifacts (e.g., fringing at 20-23 $\mu \mathrm{m}$ ). The luminosityweighted average has the steepest $30 \mu \mathrm{m}$ continuum, an indication that the most luminous sources have the warmest thermal SEDs. The 70 and $160 \mu \mathrm{m}$ matched intensities (as described in $\S 3.2$ ) are shown for each template. All five template spectra are available in the electronic edition (see Table 8).

The full sample was used to investigate the redshift response of the Spitzer bands, defined as

$$
R(z)=\frac{\int g_{\lambda} I_{\lambda}(z) d \lambda}{\int_{5 \mu \mathrm{m}}^{20 \mu \mathrm{m}} I_{\lambda}(z) d \lambda},
$$

where $g_{\lambda}$ is the effective filter transmission function for the three broadband Spitzer imaging filters of interest: IRS peakup blue $(16 \mu \mathrm{m})$ and red $(22 \mu \mathrm{m})$, and MIPS $24 \mu \mathrm{m}$. $R(z)$ quantifies the expected broadband response to a galaxy of a given spectral appearance. The normalization limits were chosen to ensure that the PAH spectrum does not suffer artificial variations due to the large range of slopes in the $20-30 \mu \mathrm{m}$ dust continuum.

In Figure $19 R(z)$ is plotted relative to its sample mean at each redshift, indicating a wide range in broad photometric response arising from normal variations in the PAH spectrum and, to a lesser degree, the underlying continuum. In the highest luminosity quartile, the range of variation is reduced by roughly $30 \%$, and there is a waist of diminished variation (a factor of 1.4) at $z \sim 0.6$, in the relatively stable rest-frame continuum between the 11 and $17 \mu \mathrm{m}$ bands. At the low- and high-redshifts ends, variations in the continuum shape dominate the response. The median range in the $10 \%-90 \%$ trimmed sample is roughly a factor of 2 (1.6 in the most luminous quartile). At redshifts where the region bracketing the 7.7 and $11.3 \mu \mathrm{m}$ features shifts through the bands, the range of variation increases significantly.

At luminosities above $L_{\mathrm{TIR}} \sim 10^{11} L_{\odot}$, other MIR emission components can begin to dominate over PAHs. In particular, the hot dust emission of embedded AGNs can contribute the bulk of the MIR power and dilute away any PAH emission present, and very strong silicate absorption can have a profound effect on the shape of the MIR continuum. A fundamental question in the modeling of deep infrared survey results is what combination of features constitutes a useful suite of galaxy template spectra. There is no guarantee that distant galaxies forming in the early universe will exhibit the same infrared properties as local galaxies, even if carefully subdivided by total infrared luminosity, metallicity, or other systemic physical properties. Because PAH emission is detected or inferred at ever higher redshifts in systems with a wide variety of infrared properties, applying the complete range of locally observed PAH behavior is the only option 


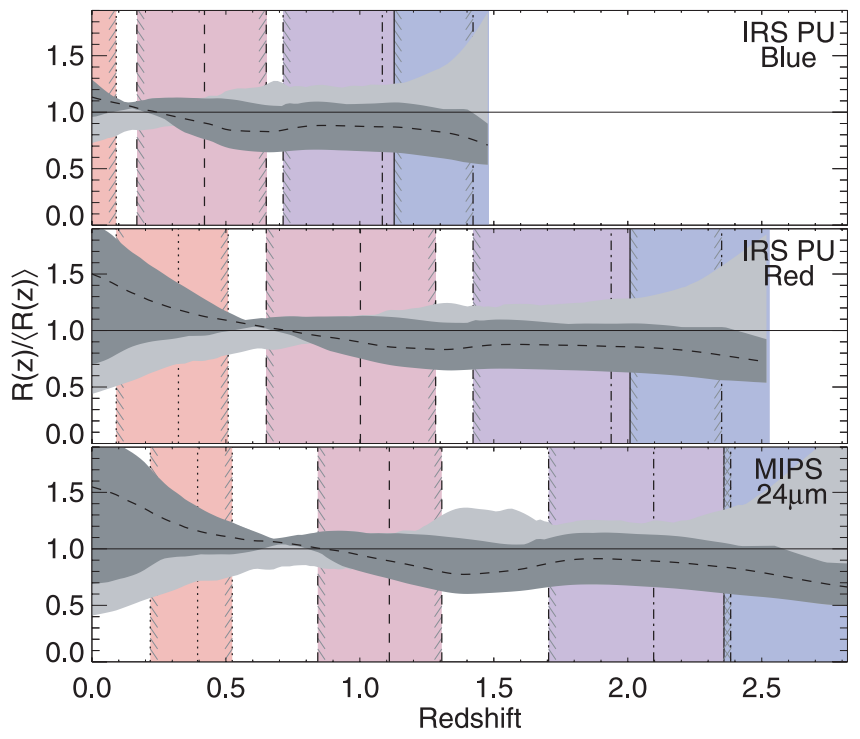

FIG. 19.- - Redshift response of three broadband Spitzer filters (IRS PU blue at $16 \mu \mathrm{m}$, IRS PU red at $22 \mu \mathrm{m}$, and MIPS $24 \mu \mathrm{m}$ ) to the range of sample spectra, relative to the mean response at each redshift. The global luminosity-weighted mean relative response is shown as the dashed line [the mean PAH template spectrum would produce the flat line $R(z)=1.0]$. The light gray region encloses the $10 \%-90 \%$ range of variation within the full sample, and the dark gray region encloses a similar $10 \%-90 \%$ range for the most luminous quartile of the sample $\left(L_{\text {TIR }}>2.6 \times 10^{10} L_{\odot}\right)$. The redshifts where the peak wavelengths of the main PAH features are shifted to the $50 \%$ power and transmission-weighted central wavelengths of each broadband filter are indicated by shaded regions for the main PAH bands at (from left to right, red to blue) $17 \mu \mathrm{m}$ (dotted line, red), $11.3 \mu \mathrm{m}$ (long-dashed line, red-blue), $7.7 \mu \mathrm{m}$ (dot-dashed line, blue-red), and $6.2 \mu \mathrm{m}$ (triple-dot-dashed line, blue).

that fully brackets the known uncertainties that result from natural variations in the PAH emission spectrum.

\section{SUMMARY AND CONCLUSIONS}

We have presented a new suite of 5-38 $\mu \mathrm{m}$ spectra from the inner $\sim \frac{1}{2} \operatorname{arcmin}^{2}$ (roughly $1-10 \mathrm{kpc}^{2}$ ) of a broadly selected sample of nearby, predominantly star-forming galaxies, many of which host weak AGNs. The spectra are dominated in most cases by PAH emission bands limited to the range 5-19 $\mu \mathrm{m}$, stellar photospheric emission of varying strength, smooth thermal dust continuum, and occasional absorption by silicate grains.

The low-resolution spectra of star-forming galaxies can be successfully reproduced using a simple physically motivated decomposition model with a small number of components, including starlight, thermal dust continuum, and line and dust emission features, all absorbed by single-parameter dust extinction. The decomposition method is also successful at recovering unresolved emission lines blended with dust features.

Weak dust emission features at 6.69 and $18.92 \mu \mathrm{m}$ are reported for the first time in extragalactic sources, and many of the weak features formerly seen only in $I S O$ spectra of one or two of the brightest starburst galaxies are found commonly among our sample. The $17 \mu \mathrm{m}$ complex correlates strongly with other PAH bands, supporting the identification of this new feature with PAH.

Compared to spline-based or other simple techniques for estimating the strengths and continuum underlying PAH features, the decomposition method recovers significantly more of the extended feature power. Spline methods agree reasonably well within a simple scalar factor for the two main bands in clean continuum regions at 6.2 and $11.3 \mu \mathrm{m}$ but recover much less of the total fea- ture power, and with significantly more scatter, for the blended complexes at 7.7 and $8.6 \mu \mathrm{m}$.

We have formulated a new extinction profile for use in the 5-38 $\mu \mathrm{m}$ region. Measurable dust absorption at MIR wavelengths by silicate grains is found to occur in $\sim \frac{1}{8}$ of the sample targets. Most normal star-forming galaxies appear to suffer less than $A_{V} \sim 3$ mag of foreground screen silicate extinction, averaged over their central few square kiloparsecs.

Matched Spitzer photometry at 24, 70, and $160 \mu \mathrm{m}$, tracing the bolometric dust emission, highlights the remarkable power emitted solely in the PAH bands, which in aggregate can contribute up to $20 \%$ of the total $3-1100 \mu \mathrm{m}$ infrared luminosity, with a typical value of $10 \%$. The PAH features vary by a factor of 10 relative to $24 \mu \mathrm{m}$, with indications that both stochastically and thermally heated grains contribute to the continuum there.

The individual PAH bands show considerable variability (factors of 2-5 in internal strength ratios in the sample), with some features varying more than others. The $17 \mu \mathrm{m}$ band in particular exhibits variation that tracks with metallicity, such that systems with higher oxygen abundance are more likely to have stronger $17 \mu \mathrm{m}$ emission relative to the shorter features. Since the longer wavelength PAH emission is expected to arise from larger PAH grains, this could indicate that the size distribution of the PAHs depends on the metal content of the ISM, even at relatively high metallicities reaching $1.5 Z_{\odot}$.

Contrary to the effects of luminous AGNs, which completely destroy PAH grains or mask their emission bands with hot dust, the presence of low-luminosity AGNs in the nucleus of a galaxy has a profound effect on the form of its PAH spectrum, leading in some cases to peculiar emission with very weak or absent 6.2, 7.7, and $8.6 \mu \mathrm{m}$ bands, suppressed in strength by up to a factor of 10. Only sample galaxies with low-luminosity LINER or Seyfert classifications from their optical nuclear spectra produce these exceedingly unusual band ratios, raising the possibility that the weak AGN itself can directly modify the PAH grain size distribution and even serve as the excitation source for PAH emission. If so, the abnormal PAH strengths could be used to detect such weak AGN systems in dusty galaxies where optical diagnostics are unavailable or unreliable. Alternatively, low star formation intensity in the centers of many AGNs may play a role in modifying the form of the PAH emission.

Both higher metallicity and the presence of an AGN shift power from the short- to long-wavelength PAH bands, most likely by rebalancing the distribution of grain sizes by favoring formation of larger grains and selectively destroying the smaller grains that emit the bulk of the shorter wavelength PAH features. The integrated luminosity of the PAH bands, relative to the total infrared, also shows an overall increase with increasing oxygen abundance, but this trend does not hold for galaxies hosting AGNs, which emit a much smaller fraction of their total infrared luminosity in the PAH bands (a few percent), even at relatively high metallicity. Both the unusual PAH spectrum and the diminished PAH luminosity in these sources caution against the direct use of the absolute PAH band strength as an indicator of star formation rate in systems known to harbor AGNs.

Taking into account a trimmed range of variations in the PAH band ratios and the shape of the MIR continuum seen in the sample, we find that, compared to the common assumption of an invariant PAH emission template used to model high-redshift sources, at least a factor of 2 variation in broadband photometric response is imparted at 16,22 , and $24 \mu \mathrm{m}$, at all redshifts $z \lesssim 2.5$. At higher total infrared luminosities, above $2.6 \times 10^{10} L_{\odot}$, the effective range is smaller by roughly $30 \%$, but still substantial. While it is likely that as galaxy luminosities approach $10^{12} L_{\odot}$, 
other effects, such as the growing contribution of obscured AGNs to the typical infrared SED, will dominate the uncertainties in modeling deep MIR source populations, utilizing a single, fixed PAH template will impart factor of 2 or larger uncertainties due solely to natural variations in the form of the MIR PAH emission spectrum.

The authors thank H. Spoon for assistance with spline-based techniques, K. Masters for early access to her updated local flow model, C. Markwardt for his indispensable IDL L-M fitting package, J. Chiar, E. Peeters, C. Papovich, D. Elbaz, and R. Chary for helpful discussions, and an anonymous referee for many useful suggestions. This work made use of the NASA/IPAC Extragalactic Database (NED), operated by JPL/Caltech, under contract with NASA. Support for this work, part of the Spitzer Space Telescope Legacy Science Program, was provided by NASA through contract 1224769 issued by JPL/Caltech under contract 1407 . B. T. D. has been partially supported by NSF grant AST 04-06883.
Allamandola, L. J., Hudgins, D. M., \& Sandford, S. A. 1999, ApJ, 511, L115 Allamandola, L. J., Tielens, A. G. G. M., \& Barker, J. R. 1985, ApJ, 290, L25 Armus, L., et al. 2004, ApJS, 154, 178

Barvainis, R., Antonucci, R., \& Helou, G. 1999, AJ, 118, 645

Beintema, D. A., et al. 1996, A\&A, 315, L369

Boulanger, F., Boisssel, P., Cesarsky, D., \& Ryter, C. 1998, A\&A, 339, 194

Brandl, B. R., et al. 2006, ApJ, 653, 1129

Bregman, J., \& Temi, P. 2005, ApJ, 621, 831

Bregman, J. D., Bregman, J. N., \& Temi, P. 2006, preprint (astro-ph/0604369)

Bressan, A., et al. 2006, ApJ, 639, L55

Calzetti, D., et al. 2005, ApJ, 633, 871

Cannon, J. M., et al. 2006, ApJ, 647, 293

Caputi, K. I., et al. 2006, ApJ, 637, 727

Cesarsky, D., Lequeux, J., Abergel, A., Perault, M., Palazzi, E., Madden, S., \& Tran, D. 1996, A\&A, 315, L305

Cesarsky, D., Lequeux, J., Pagani, L., Ryter, C., Loinard, L., \& Sauvage, M. 1998, A\&A, 337, L35

Chiar, J. E., \& Tielens, A. G. G. M. 2006, ApJ, 637, 774

Contursi, A., et al. 2000, A\&A, 362, 310

Dale, D. A., \& Helou, G. 2002, ApJ, 576, 159

Dale, D. A., Helou, G., Contursi, A., Silbermann, N. A., \& Kolhatkar, S. 2001, ApJ, 549, 215

Dale, D. A., et al. 2005, ApJ, 633, 857 2006, ApJ, 646, 161

Draine, B. T., \& Anderson, N. 1985, ApJ, 292, 494

Draine, B. T., \& Li, A. 2001, ApJ, 551, 807 2006, preprint (astro-ph/0608003)

Elbaz, D., Cesarsky, C. J., Chanial, P., Aussel, H., Franceschini, A., Fadda, D., \& Chary, R. R. 2002, A\&A, 384, 848

Engelbracht, C. W., Gordon, K. D., Rieke, G. H., Werner, M. W., Dale, D. A., \& Latter, W. B. 2005, ApJ, 628, L29

Fitzpatrick, E. L., \& Massa, D. 1986, ApJ, 307, 286

Genzel, R., et al. 1998, ApJ, 498, 579

Gillett, F. C., Forrest, W. J., \& Merrill, K. M. 1973, ApJ, 183, 87

Guhathakurta, P., \& Draine, B. T. 1989, ApJ, 345, 230

Hao, L., et al. 2005, ApJ, 625, L75

Helou, G., Lu, N. Y., Werner, M. W., Malhotra, S., \& Silbermann, N. 2000, ApJ, 532, L21

Helou, G., et al. 2004, ApJS, 154, 253

Ho, L. C., Filippenko, A. V., \& Sargent, W. L. 1995, ApJS, 98, 477

Ho, L. C., Filippenko, A. V., \& Sargent, W. L. W. 1997, ApJ, 487, 579

Hogg, D. W., Tremonti, C. A., Blanton, M. R., Finkbeiner, D. P., Padmanabhan, N., Quintero, A. D., Schlegel, D. J., \& Wherry, N. 2005, ApJ, 624, 162

Hony, S., Van Kerckhoven, C., Peeters, E., Tielens, A. G. G. M., Hudgins, D. M., \& Allamandola, L. J. 2001, A\&A, 370, 1030

Houck, J. R., et al. 2004, ApJS, 154, 18 2005, ApJ, 622, L105

Huang, J., et al. 2006, preprint

Joblin, C., Tielens, A. G. G. M., Geballe, T. R., \& Wooden, D. H. 1996, ApJ, 460, L119

Kaneda, H., Onaka, T., \& Sakon, I. 2005, ApJ, 632, L83

Kauffmann, G., et al. 2003, MNRAS, 346, 1055

Kemper, F., Vriend, W. J., \& Tielens, A. G. G. M. 2004, ApJ, 609, 826

Kennicutt, R. C., Jr., et al. 2003, PASP, 115, 928

Kewley, L. J., Groves, B., Kauffmann, G., \& Heckman, T. 2006, MNRAS, 372, 961

Lagache, G., et al. 2004, ApJS, 154, 112

Laurent, O., Mirabel, I. F., Charmandaris, V., Gallais, P., Madden, S. C., Sauvage, M., Vigroux, L., \& Cesarsky, C. 2000, A\&A, 359, 887

Le Floc'h, E., et al. 2005, ApJ, 632, 169

Léger, A., D’Hendecourt, L., Boissel, P., \& Désert, F. X. 1989, A\&A, 213, 351

Leger, A., \& Puget, J. L. 1984, A\&A, 137, L5
Leitherer, C., et al. 1999, ApJS, 123, 3

Li, A. 2004, in ASP Conf. Ser. 309, Astrophysics of Dust, ed. A. N. Witt, G. C. Clayton, \& B. T. Draine (San Francisco: ASP), 417

Li, A., \& Draine, B. T. 2001, ApJ, 554, 778

Lu, N., et al. 2003, ApJ, 588, 199

Lutz, D., Spoon, H. W. W., Rigopoulou, D., Moorwood, A. F. M., \& Genzel, R. 1998, ApJ, 505, L103

Lutz, D., Valiante, E., Sturm, E., Genzel, R., Tacconi, L. J., Lehnert, M. D., Sternberg, A., \& Baker, A. J. 2005, ApJ, 625, L83

Madden, S. C., Galliano, F., Jones, A. P., \& Sauvage, M. 2006, A\&A, 446, 877

Martín-Hernández, N. L., Vermeij, R., Tielens, A. G. G. M., van der Hulst, J. M., \& Peeters, E. 2002, A\&A, 389, 286

Meijerink, R., \& Spaans, M. 2005, A\&A, 436, 397

Moutou, C., Verstraete, L., Léger, A., Sellgren, K., \& Schmidt, W. 2000, A\&A, 354, L17

O'Halloran, B., Satyapal, S., \& Dudik, R. P. 2006, ApJ, 641, 795

Papovich, C., et al. 2004, ApJS, 154, 70

. 2006, ApJ, 640, 92

Peeters, E., Hony, S., Van Kerckhoven, C., Tielens, A. G. G. M., Allamandola, L. J., Hudgins, D. M., \& Bauschlicher, C. W. 2002, A\&A, 390, 1089

Peeters, E., Mattioda, A. L., Hudgins, D. M., \& Allamandola, L. J. 2004, ApJ, 617, L65

Pilyugin, L. S., \& Thuan, T. X. 2005, ApJ, 631, 231

Puget, J. L., Léger, A., \& Boulanger, F. 1985, A\&A, 142, L19

Reddy, N. A., Steidel, C. C., Fadda, D., Yan, L., Pettini, M., Shapley, A. E., Erb, D. K., \& Adelberger, K. L. 2006, ApJ, 644, 792

Rieke, G. H., \& Lebofsky, M. J. 1985, ApJ, 288, 618

Rieke, G. H., et al. 2004, ApJS, 154, 25

Rigopoulou, D., Spoon, H. W. W., Genzel, R., Lutz, D., Moorwood, A. F. M., \& Tran, Q. D. 1999, AJ, 118, 2625

Rosenberg, J. L., Ashby, M. L. N., Salzer, J. J., \& Huang, J.-S. 2006, ApJ, 636, 742 Roussel, H., et al. 2006, ApJ, 646, 841

Schutte, W. A., Tielens, A. G. G. M., \& Allamandola, L. J. 1993, ApJ, 415, 397 Siebenmorgen, R., Haas, M., Krügel, E., \& Schulz, B. 2005, A\&A, 436, L5

Silva, L., Granato, G. L., Bressan, A., \& Danese, L. 1998, ApJ, 509, 103

Smith, J. D. T., et al. 2004, ApJS, 154, 199

Spoon, H. W. W., et al. 2004, ApJS, 154, 184 2006, ApJ, 638, 759

Sturm, E., Lutz, D., Tran, D., Feuchtgruber, H., Genzel, R., Kunze, D., Moorwood, A. F. M., \& Thornley, M. D. 2000, A\&A, 358, 481

Sturm, E., et al. 1996, A\&A, 315, L133 2005, ApJ, 629, L21

Thornley, M. D., Schreiber, N. M. F., Lutz, D., Genzel, R., Spoon, H. W. W., Kunze, D., \& Sternberg, A. 2000, ApJ, 539, 641

Uchida, K. I., Sellgren, K., Werner, M. W., \& Houdashelt, M. L. 2000, ApJ, 530,817

Van Kerckhoven, C., et al. 2000, A\&A, 357, 1013

Vermeij, R., Peeters, E., Tielens, A. G. G. M., \& van der Hulst, J. M. 2002, A\&A, 382, 1042

Verstraete, L., Puget, J. L., Falgarone, E., Drapatz, S., Wright, C. M., \& Timmermann, R. 1996, A\&A, 315, L337

Verstraete, L., et al. 2001, A\&A, 372, 981

Voit, G. M. 1992, MNRAS, 258, 841

Waters, L. B. F. M., et al. 1998, A\&A, 331, L61

Webb, T. M. A., et al. 2006, ApJ, 636, L17

Weingartner, J. C., \& Draine, B. T. 2001, ApJS, 134, 263

Werner, M. W., Uchida, K. I., Sellgren, K., Marengo, M., Gordon, K. D., Morris, P. W., Houck, J. R., \& Stansberry, J. A. 2004, ApJS, 154, 309

Wu, Y., Charmandaris, V., Hao, L., Brandl, B. R., Bernard-Salas, J., Spoon,

H. W. W., \& Houck, J. R. 2006, ApJ, 639, 157

Yan, L., et al. 2004, ApJS, 154, 60

. 2005, ApJ, 628, 604 